\title{
SUPERCONVERGENT DISCONTINUOUS GALERKIN METHODS FOR SECOND-ORDER ELLIPTIC PROBLEMS
}

\author{
BERNARDO COCKBURN, JOHNNY GUZMÁN, AND HAIYING WANG
}

\begin{abstract}
We identify discontinuous Galerkin methods for second-order elliptic problems in several space dimensions having superconvergence properties similar to those of the Raviart-Thomas and the Brezzi-Douglas-Marini mixed methods. These methods use polynomials of degree $k \geq 0$ for both the potential as well as the flux. We show that the approximate flux converges in $L^{2}$ with the optimal order of $k+1$, and that the approximate potential and its numerical trace superconverge, in $L^{2}$-like norms, to suitably chosen projections of the potential, with order $k+2$. We also apply element-by-element postprocessing of the approximate solution to obtain new approximations of the flux and the potential. The new approximate flux is proven to have normal components continuous across inter-element boundaries, to converge in $L^{2}$ with order $k+1$, and to have a divergence converging in $L^{2}$ also with order $k+1$. The new approximate potential is proven to converge with order $k+2$ in $L^{2}$. Numerical experiments validating these theoretical results are presented.
\end{abstract}

\section{INTRODUCTION}

In this paper, we uncover a large class of discontinuous Galerkin (DG) methods for the following second-order elliptic problem:

$$
\begin{aligned}
\boldsymbol{c} \boldsymbol{q}+\nabla u & =0 & & \text { in } \Omega, \\
\nabla \cdot \boldsymbol{q} & =f & & \text { in } \Omega, \\
u & =g & & \text { on } \partial \Omega_{D}, \\
\boldsymbol{q} \cdot \boldsymbol{n} & =\text { q }_{\mathrm{N}} & & \text { on } \partial \Omega_{N},
\end{aligned}
$$

with optimal convergence properties for the approximate flux and superconvergence properties similar to those of the classical mixed methods of Raviart-Thomas (RT) [15 and Brezzi-Douglas-Marini (BDM) 5. Here $\Omega \subset \mathbb{R}^{d}$ is a polyhedral domain $(d \geq 2), f \in L^{2}(\Omega)$, and $\boldsymbol{c}=\boldsymbol{c}(\boldsymbol{x})$ is a symmetric $d \times d$ matrix function that is uniformly positive definite on $\Omega$ with components in $L^{\infty}(\Omega)$. Some of our results require the domain $\Omega$ to be convex.

Received by the editor October 9, 2007 and, in revised form, January 31, 2008.

2000 Mathematics Subject Classification. Primary 65M60, 65N30, 35L65.

Key words and phrases. Finite element methods, mixed methods, discontinuous Galerkin methods, superconvergence, postprocessing.

B. Cockburn was supported in part by the National Science Foundation (Grant DMS-0712955) and by the University of Minnesota Supercomputing Institute.

J. Guzmán was supported by a National Science Foundation Mathematical Science Postdoctoral Research Fellowship (DMS-0503050). 
To describe our results, we need to introduce some notation. We denote by $\Omega_{h}=\{K\}$ a triangulation of the domain $\Omega$ of shape-regular simplices $K$ and set $\partial \Omega_{h}:=\left\{\partial K: K \in \Omega_{h}\right\}$. We associate to this triangulation the set of interior faces $\mathscr{E}_{h}^{i}$ and the set of boundary faces $\mathscr{E}_{h} \partial$. We say that $e \in \mathscr{E}_{h}^{i}$ if there are two simplices $K^{+}$and $K^{-}$in $\Omega_{h}$ such that $e=\partial K^{+} \cap \partial K^{-}$, and we say that $e \in \mathscr{E}_{h}^{\partial}$ if there is a simplex in $\Omega_{h}$ such that $e=\partial K \cap \partial \Omega$. We set $\mathscr{E}_{h}:=\mathscr{E}_{h} i \cup \mathscr{E}_{h} \partial$.

We assume that the approximate solution $\left(\boldsymbol{q}_{h}, u_{h}\right) \in \boldsymbol{V}_{h} \times W_{h}$ satisfies the formulation

$$
\begin{aligned}
& \left(\boldsymbol{c} \boldsymbol{q}_{h}, \boldsymbol{v}\right)_{\Omega_{h}}-\left(u_{h}, \nabla \cdot \boldsymbol{v}\right)_{\Omega_{h}}+\left\langle\widehat{u}_{h}, \boldsymbol{v} \cdot \boldsymbol{n}\right\rangle_{\partial \Omega_{h}}=0, \\
& -\left(\boldsymbol{q}_{h}, \nabla \omega\right)_{\Omega_{h}}+\left\langle\widehat{\boldsymbol{q}}_{h} \cdot \boldsymbol{n}, \omega\right\rangle_{\partial \Omega_{h}}=(f, \omega)_{\Omega_{h}},
\end{aligned}
$$

for all $(\boldsymbol{v}, \omega) \in \mathbf{V}_{h} \times \mathbf{W}_{h}$, where

$$
\widehat{u}_{h} \text { is single-valued on } \mathscr{E}_{h} \text {, }
$$$$
\text { the normal component of } \widehat{\boldsymbol{q}}_{h} \text { is single-valued on } \mathscr{E}_{h} \text {, }
$$

and

$$
\begin{array}{ll}
\widehat{u}_{h}=g & \text { on } \partial \Omega_{D}, \\
\widehat{\boldsymbol{q}}_{h} \cdot \boldsymbol{n}=\mathbf{q}_{\mathrm{N}} & \text { on } \partial \Omega_{N} .
\end{array}
$$

Here, we have used the notation

$$
\begin{aligned}
(\boldsymbol{\sigma}, \boldsymbol{v})_{\Omega_{h}} & :=\sum_{i=1}^{d}\left(\sigma_{i}, v_{i}\right)_{\Omega_{h}} & & \forall \boldsymbol{\sigma}, \boldsymbol{v} \in \boldsymbol{H}^{1}\left(\Omega_{h}\right), \\
(\zeta, \omega)_{\Omega_{h}} & :=\sum_{K \in \Omega_{h}} \int_{K} \zeta(x) \omega(x) d x, & & \forall \zeta, \omega \in H^{1}\left(\Omega_{h}\right), \\
\langle\boldsymbol{v} \cdot \boldsymbol{n}, \mu\rangle_{\partial \Omega_{h}}: & =\sum_{K \in \Omega_{h}} \int_{\partial K} \boldsymbol{v}(\gamma) \cdot \boldsymbol{n} \mu(\gamma) d \gamma & & \forall(\boldsymbol{v}, \mu) \in \boldsymbol{L}^{2}\left(\partial \Omega_{h}\right) \times L^{2}\left(\partial \Omega_{h}\right) .
\end{aligned}
$$

The outward normal unit vector to $\partial K$ is denoted by $\boldsymbol{n}$. Finally, if $S$ represents a given space, we are writing $\boldsymbol{S}\left(\Omega_{h}\right):=\left[S\left(\Omega_{h}\right)\right]^{d}$ and

$$
\begin{aligned}
S\left(\Omega_{h}\right) & :=\left\{\omega: \Omega_{h} \mapsto \mathbb{R},\left.\omega\right|_{K} \in S(K) \forall K \in \Omega_{h}\right\}, \\
S\left(\partial \Omega_{h}\right) & :=\left\{\omega: \partial \Omega_{h} \mapsto \mathbb{R},\left.\omega\right|_{\partial K} \in S(\partial K) \forall K \in \Omega_{h}\right\} .
\end{aligned}
$$

Note that we are not assuming that the approximate solution under consideration is determined by the above equations, but only that it satisfies them; see, for example, the equations defining the approximate solution of the discontinuous Petrov-Galerkin method 4 .

We are now ready to discuss our results. For a long while, no DG method using the choice of spaces

$$
\boldsymbol{V}_{h} \times W_{h}=\mathbf{V}_{h} \times \mathbf{W}_{h}=\mathbf{V}_{h}^{k} \times \mathbf{W}_{h}^{k},
$$

where

$$
\begin{array}{rll}
\mathbf{V}_{h}^{k}=\left\{\boldsymbol{v} \in L^{2}(\Omega):\right. & \left.\boldsymbol{v}\right|_{K} \in \mathcal{P}^{k}(K) & \left.\forall K \in \Omega_{h}\right\}, \\
\mathbf{W}_{h}^{k}=\left\{w \in L^{2}(\Omega):\right. & \left.w\right|_{K} \in \mathcal{P}^{k}(K) & \left.\forall K \in \Omega_{h}\right\}
\end{array}
$$

was known to provide an approximate flux $\boldsymbol{q}_{h}$ converging with the optimal order $k+1$; here, $\mathcal{P}^{k}(K)$ stands for the set of polynomials of degree at most $k$ defined on $K$, 
and $\mathcal{P}^{k}(K)$ for $\mathbb{R}^{d}$-valued functions whose components belong to $\mathcal{P}^{k}(K)$. Indeed, in the unified analysis of DG methods for second-order elliptic problems, 2], it was shown that the approximations $u_{h}$ and $\boldsymbol{q}_{h}$ given by consistent and stable DG methods using the spaces (1.3) converge in $L^{2}$ with order $k+1$ and $k$, respectively, for any $k \geq 1$; see also [7, where the performances of representative DG methods are compared.

In 8, DG methods using the spaces (1.3), which do not fit in the abovementioned unified analysis, were thoroughly studied. Those methods are obtained when the numerical traces on $\mathscr{E}_{h}^{i}$ are chosen as follows:

$$
\begin{aligned}
& \widehat{u}_{h}=\left\{\left\{u_{h}\right\}\right\}-\boldsymbol{C}_{12} \cdot \llbracket u_{h} \rrbracket+C_{22} \llbracket \boldsymbol{q}_{h} \rrbracket, \\
& \widehat{\boldsymbol{q}}_{h}=\left\{\left\{\boldsymbol{q}_{h}\right\}\right\}+\boldsymbol{C}_{12} \cdot \llbracket \boldsymbol{q}_{h} \rrbracket+C_{11} \llbracket u_{h} \rrbracket,
\end{aligned}
$$

and the penalization parameter $C_{22}$ is taken to be strictly positive. We are using the standard notation for the averages $\{[\cdot\}$ and the jumps $\llbracket \cdot \rrbracket$; see $[2]$.

For these methods, it was shown 8 that if $C_{12}$ is of order one, and $C_{11}$ and $1 / C_{22}$ are of order $1 / h$, then $u_{h}$ and $\boldsymbol{q}_{h}$ converge in $L^{2}$ with order $k+1$ and $k$, respectively, for any $k \geq 1$. The same orders of convergence were proven to hold if we set $C_{22}=0$, that is, for the so-called local DG (LDG ) method; see also [2].

Better orders of convergence were obtained for the approximate flux if $C_{11}$ is decreased and $C_{22}$ is increased. Indeed, it was shown [8] that if $C_{11}$ and $C_{22}$ are of order one, then $u_{h}$ and $\boldsymbol{q}_{h}$ converge in $L^{2}$ with order $k+1$ and $k+1 / 2$, respectively, for any $k \geq 0$. In spite of this better convergence property, these DG methods have not been paid too much attention since they are very difficult to implement (precisely because $\left.C_{22} \neq 0\right)$. On the other hand, in [12, an LDG method $\left(C_{22}=\right.$ 0 ) with those same orders of convergence was obtained which employed $C_{11}$ of order one; it used Cartesian grids, tensor-product polynomial local spaces and a special choice for $\boldsymbol{C}_{12}$. Until recently, no DG method was known which provided an approximate flux converging with order $k+1$. The first DG method with such a property, the SF-H method, was recently identified in 9 .

The SF-H method is one of the local DG-hybridizable (LDG-H) methods introduced in [10. The LDG-H methods are characterized by the fact that their numerical traces on any given interior face are given by

$$
\begin{aligned}
& \widehat{u}_{h}=\left(\frac{\tau^{+}}{\tau^{-}+\tau^{+}}\right) u_{h}^{+}+\left(\frac{\tau^{-}}{\tau^{-}+\tau^{+}}\right) u_{h}^{-}+\left(\frac{1}{\tau^{-}+\tau^{+}}\right) \llbracket \boldsymbol{q}_{h} \rrbracket, \\
& \widehat{\boldsymbol{q}}_{h}=\left(\frac{\tau^{-}}{\tau^{-}+\tau^{+}}\right) \boldsymbol{q}_{h}^{+}+\left(\frac{\tau^{+}}{\tau^{-}+\tau^{+}}\right) \boldsymbol{q}_{h}^{-}+\left(\frac{\tau^{+} \tau^{-}}{\tau^{-}+\tau^{+}}\right) \llbracket u_{h} \rrbracket,
\end{aligned}
$$

where $\tau^{ \pm}$are nonnegative constants. The main interest of these methods is that they can be implemented very efficiently in spite of the fact that in this case the coefficient $C_{22}=1 /\left(\tau^{-}+\tau^{+}\right)$is different from zero; see [10. The distinctive feature of the SF-H method is that on each simplex $K, \tau$ is equal to zero except on a single, arbitrarily chosen face of $K$. Thanks to this choice, it is possible to show [9] optimal convergence properties, for the approximate flux, as well as convergence of order $k+2$ of the approximate potential, and its numerical trace superconverge to some projections of the potential. As a consequence, a postprocessing of the approximate solution, $u_{h}^{\star}$, could be constructed which provides an approximation of the potential converging with order $k+2$ in $L^{2}$. 
The fact that similar properties hold for the hybridized version of the RT and that of the BDM mixed methods is no coincidence. In fact, the SF-H method was actually discovered by exploiting the similarities that hybridizable DG methods and the hybridized versions of classical mixed methods have, as made evident in the framework proposed in [10]. Since, thanks to that framework, the SF-H could be seen as a DG method in between the RT and BDM methods, an extension of the analysis of the hybridized versions of the RT and BDM method was used to (both devise and) analyze the SF-H method and to obtain for it the above-mentioned convergence and superconvergence properties.

In this paper, however, we do not exploit the hybridization framework introduced in [10]. Instead, we rely on an approach based on trying to rewrite the general formulation (1.2) as that of the Raviart-Thomas method. This approach was introduced in the study of the local conservativity properties of the continuous Galerkin method; see [11. In our setting, this approach allows us to conclude that the convergence and superconvergence properties under consideration hold for any method satisfying the formulation (1.2) when, roughly speaking, the normal component of $\widehat{\boldsymbol{q}}_{h}-\boldsymbol{q}_{h}$ is small enough, or, when the approximate flux $\boldsymbol{q}_{h}$ is close to being in $H(\operatorname{div}, \Omega)$. We then prove that this condition is satisfied by DG (1.4) and LDG-H (1.5) methods for several choices of the parameters defining their numerical traces. We thus refine the error estimates obtained for some of the DG methods considered in [8, and extend the results obtained for the SF-H method in [9] to a large class of LDG-H methods. In Table 1, we denote by $e_{K}^{\tau}$ the face of the simplex $K$ at which $\tau$ is a maximum.

TABLE 1. Examples of conditions guaranteeing the optimal convergence of $\boldsymbol{q}_{h}$ and the superconvergence properties of $u_{h}$ and $\widehat{u}_{h}$.

\begin{tabular}{ccc}
\hline method & positivity condition & boundedness condition \\
\hline \hline DG & $C_{11}, C_{22}>0$ & $C_{11},\left|\boldsymbol{C}_{12}\right|$ bounded \\
LDG-H & $\tau \geq 0$ & and $C_{11} \sim 1 / C_{22}$ \\
& $\left.\tau\right|_{\partial K \backslash e_{K}^{\tau}}$ bounded \\
\hline
\end{tabular}

The paper is organized as follows. In Section 2 we state and discuss our two main results, namely, the optimal convergence of the approximate flux, Theorem 2.1, and the superconvergence properties of $u_{h}$ and $\widehat{u}_{h}$, Theorem 2.3. We also introduce the postprocessed approximations $\boldsymbol{q}_{h}^{\star}$ and $u_{h}^{\star}$ and obtain optimal convergence results for them; see Theorems 2.2 and 2.4, respectively. All these results are proved in full detail in Section 4. In Section 3, we provide several examples of methods for which these theorems hold, and in Section 5. we give numerical evidence confirming the theoretical predictions. We end in Section [6 by sketching some extensions and by providing some concluding remarks.

\section{THE MAIN RESULtS}

In all the theorems of this section, we only assume that the following inclusion conditions hold on the spaces of test functions:

$$
\begin{gathered}
\mathbf{V}_{h} \supset \mathbf{V}_{h}^{k}, \\
\mathbf{W}_{h} \supset \mathbf{W}_{h}^{k},
\end{gathered}
$$


for some nonnegative integer $k$. No condition is imposed on the spaces of trial functions $\boldsymbol{V}_{h}$ and $W_{h}$.

2.1. Optimal convergence of $\boldsymbol{q}_{h}$. To state our results, we need to introduce some notation. Given a function $\boldsymbol{\sigma} \in H\left(\operatorname{div}, \Omega_{h}\right)$ and an arbitrary simplex $K \in \Omega_{h}$, the restriction of $\boldsymbol{\Pi}_{\ell}^{\mathrm{RT}} \boldsymbol{\sigma}$ on $K$ is defined as the only element of $\mathcal{P}^{\ell}(K)+\boldsymbol{x} \mathcal{P}^{\ell}(K)$ satisfying

$$
\begin{array}{rlrl}
\left\langle\left(\boldsymbol{\Pi}_{\ell}^{\mathrm{RT}} \boldsymbol{\sigma}-\boldsymbol{\sigma}\right) \cdot \boldsymbol{n}, \mu\right\rangle_{e} & =0 & & \forall \mu \in \mathcal{P}^{\ell}(e) \text { for all faces } e \text { of } K, \\
\left(\boldsymbol{\Pi}_{\ell}^{\mathrm{RT}} \boldsymbol{\sigma}-\boldsymbol{\sigma}, \boldsymbol{v}\right)_{K}=0 & \forall \boldsymbol{v} \in \mathcal{P}^{\ell-1}(K) .
\end{array}
$$

Given a function $\eta \in L^{2}\left(\partial \Omega_{h}\right)$, and an arbitrary simplex $K \in \Omega_{h}$, the restriction of $\mathrm{P}_{\partial}^{\ell} \eta$ to a face $e$ of $K$ is defined as the element of $\mathcal{P}^{\ell}(e)$ that satisfies

$$
\left\langle\mathrm{P}_{\partial}^{\ell} \eta-\eta, \mu\right\rangle_{e}=0, \quad \forall \mu \in \mathcal{P}^{\ell}(e) .
$$

Note that, on the interior faces, $\mathrm{P}_{\partial}^{\ell} \eta$ is in general double-valued. Finally, we set, for any $\boldsymbol{\tau} \in \boldsymbol{L}^{2}\left(\partial \Omega_{h}\right)$,

$$
\boldsymbol{\|} \boldsymbol{\tau} \|_{L^{2}\left(\mathscr{E}_{h} ; h\right)}:=\left(\sum_{K \in \Omega_{h}} h_{K}\|\boldsymbol{\tau} \cdot \boldsymbol{n}\|_{L^{2}(\partial K)}^{2}\right)^{1 / 2} .
$$

We are now ready to state the first of our main results.

Theorem 2.1. For any method of the form (1.2) satisfying the inclusions (2.6) for $k \geq 0$, we have

$$
\left\|\boldsymbol{q}-\boldsymbol{q}_{h}\right\|_{L^{2}\left(\Omega_{h}\right)} \leq C\left(\left\|\boldsymbol{q}-\boldsymbol{\Pi}_{k}^{\mathrm{RT}} \boldsymbol{q}\right\|_{L^{2}(\Omega)}+\Theta_{k}\right),
$$

where

$$
\Theta_{k}=\left\|\boldsymbol{q}_{h}-\boldsymbol{\Pi}_{k}^{\mathrm{RT}} \boldsymbol{q}_{h}\right\|_{L^{2}(\Omega)}+\mid \mathrm{P}_{\partial}^{k}\left(\widehat{\boldsymbol{q}}_{h}-\boldsymbol{q}_{h}\right) \|_{L^{2}\left(\mathscr{E}_{h} ; h\right)} .
$$

Note that if the approximate flux $\boldsymbol{q}_{h}$ is given by the RT method, then $\Theta_{k}=0$ and the above result gives the classic error estimate. Note also that if $\boldsymbol{V}_{h}$ is included in the space of fluxes of the corresponding RT method, $\boldsymbol{q}_{h}=\boldsymbol{\Pi}_{k}^{\mathrm{RT}} \boldsymbol{q}_{h}$, and

$$
\Theta_{k}=\| \mathbf{P}_{\partial}^{k}\left(\widehat{\boldsymbol{q}}_{h}-\boldsymbol{q}_{h}\right) \boldsymbol{|}_{L^{2}\left(\mathscr{E}_{h} ; h\right)} .
$$

Thus, optimal convergence properties for $\boldsymbol{q}_{h}$ can be obtained provided the normal component of $\widehat{\boldsymbol{q}}_{h}-\boldsymbol{q}_{h}$ is small enough, that is, provided $\boldsymbol{q}_{h}$ is "close" to being in $H(\operatorname{div}, \Omega)$.

We can easily compute a new approximation of the flux, $\boldsymbol{q}_{h}^{\star}$, which converges with the same order as $\boldsymbol{q}_{h}$ and belongs to the space $H(\operatorname{div}, \Omega)$. This new approximate flux is obtained in an element-by-element fashion by using a slight modification of the Raviart-Thomas projection we describe next. It has been used in the framework of Darcy's flow, see [3], and also for the Navier-Stokes equations, see [13.

On each simplex $K \in \Omega_{h}$, we define the function $\boldsymbol{q}_{h}^{\star}$ as the only element of $\mathcal{P}^{k}(K)+\boldsymbol{x} \mathcal{P}^{k}(K)$ satisfying

$$
\begin{array}{rlrl}
\left\langle\left(\boldsymbol{q}_{h}^{\star}-\widehat{\boldsymbol{q}}_{h}\right) \cdot \boldsymbol{n}, \mu\right\rangle_{e} & =0 & & \forall \mu \in \mathcal{P}^{k}(e) \text { for all faces } e \text { of } K, \\
\left(\boldsymbol{q}_{h}^{\star}-\boldsymbol{q}_{h}, \boldsymbol{v}\right)_{K} & =0 & \forall \boldsymbol{v} \in \mathcal{P}^{k-1}(K) .
\end{array}
$$


Given a function $\zeta \in L^{2}(\Omega)$ and an arbitrary simplex $K \in \Omega_{h}$, the restriction of $\mathrm{P}^{\ell} \zeta$ to $K$ is defined as the element of $\mathcal{P}^{\ell}(K)$ that satisfies

$$
\left(\mathrm{P}^{\ell} \zeta-\zeta, \omega\right)_{K}=0, \quad \forall \omega \in \mathcal{P}^{\ell}(K) .
$$

The main properties of the new approximation $\boldsymbol{q}_{h}^{\star}$ are gathered in the following result.

Theorem 2.2. For any method of the form (1.2) satisfying the inclusions (2.6) for $k \geq 1$, we have that

$$
\begin{aligned}
\boldsymbol{q}_{h}^{\star} \in H(\operatorname{div}, \Omega), & \\
\left\|\boldsymbol{q}-\boldsymbol{q}_{h}^{\star}\right\|_{L^{2}(\Omega)} & \leq C\left(\left\|\boldsymbol{q}-\mathbf{\Pi}_{k}^{\mathrm{RT}} \boldsymbol{q}\right\|_{L^{2}(\Omega)}+\Theta_{k}\right), \\
\left\|\nabla \cdot\left(\boldsymbol{q}-\boldsymbol{q}_{h}^{\star}\right)\right\|_{L^{2}(\Omega)} & =\left\|f-\mathrm{P}^{k} f\right\|_{L^{2}\left(\Omega_{h}\right)} .
\end{aligned}
$$

Note that this last inequality implies that

$$
\left\|\nabla \cdot\left(\boldsymbol{q}-\boldsymbol{q}_{h}^{\star}\right)\right\|_{L^{2}(\Omega)} \leq C h^{k+1}|f|_{H^{k+1}\left(\Omega_{h}\right)}
$$

provided $f \in H^{k+1}\left(\Omega_{h}\right)$.

2.2. Superconvergence of $u_{h}, \widehat{u}_{h}$. To state the results of this subsection, we need to introduce some notation and a key hypothesis on the domain. We define $(\boldsymbol{\psi}, \phi)$ to be the solution of the so-called adjoint problem

$$
\begin{aligned}
\boldsymbol{c} \boldsymbol{\psi}+\nabla \phi=0 & \text { in } \Omega, \\
\nabla \cdot \boldsymbol{\psi}=\theta & \text { in } \Omega, \\
\phi=0 & \text { on } \partial \Omega_{D}, \\
\boldsymbol{\psi} \cdot \boldsymbol{n}=0 & \text { on } \partial \Omega_{N} .
\end{aligned}
$$

We assume that the following elliptic regularity result holds:

$$
\|\boldsymbol{\psi}\|_{\boldsymbol{H}^{r+1}\left(\Omega_{h}\right)}+\|\nabla \phi\|_{\boldsymbol{H}^{r+1}\left(\Omega_{h}\right)} \leq \mathrm{C}_{e r}\|\theta\|_{H^{r}(\Omega)},
$$

for $r \in[0, r]$. If the domain $\Omega$ is convex, this property holds for $r=0$.

Finally, we set, for any $\eta \in L^{2}\left(\partial \Omega_{h}\right)$,

$$
|\eta|_{L^{2}\left(\mathscr{E}_{h} ; h\right)}:=\left(\sum_{K \in \Omega_{h}} h_{K}\|\eta\|_{L^{2}(\partial K)}^{2}\right)^{1 / 2}
$$

We are now ready to state our second main result.

Theorem 2.3. Assume that $\Omega$ is such that the elliptic regularity result (2.12) holds with $r=0$. Then, for any method in the general form (1.2) satisfying the inclusions (2.6) for $k \geq 1$, we have

$$
\begin{aligned}
\left\|\mathrm{P}^{k-1}\left(u-u_{h}\right)\right\|_{L^{2}\left(\Omega_{h}\right)} & \leq C h\left(\left\|\boldsymbol{q}-\boldsymbol{\Pi}_{k}^{\mathrm{RT}} \boldsymbol{q}\right\|_{L^{2}(\Omega)}+\Theta_{k}\right), \\
\left|\mathrm{P}_{\partial}^{k}\left(u-\widehat{u}_{h}\right)\right|_{L^{2}\left(\mathscr{E}_{h} ; h\right)} & \leq C h\left(\left\|\boldsymbol{q}-\boldsymbol{\Pi}_{k}^{\mathrm{RT}} \boldsymbol{q}\right\|_{L^{2}(\Omega)}+\Theta_{k}\right) .
\end{aligned}
$$

As was shown in [1] for the RT method (and in [5] for the BDM method), the above superconvergence results can be used to obtain a new approximation for $u$, $u_{h}^{\star}$, which converges faster than $u_{h}$. To construct it, we use a slight modification introduced in [9] of the postprocessing proposed in [16, 17] and [14]. 
On the simplex $K$, we define the new approximation of $u, u_{h}^{\star}$, as the function of $\mathcal{P}^{k+1}(K)$ given by

$$
u_{h}^{\star}=\widetilde{u}_{h}+\frac{1}{|K|} \int_{K} u_{h} d x
$$

where $\widetilde{u}_{h}$ is the polynomial in $\mathcal{P}_{0}^{k+1}(K)$ satisfying

$$
\left(\boldsymbol{a} \nabla \widetilde{u}_{h}, \nabla w\right)_{K}=(f, w)_{K}-\left\langle w, \widehat{\boldsymbol{q}}_{h} \cdot \boldsymbol{n}\right\rangle_{\partial K} \quad \forall w \in \mathcal{P}_{0}^{k+1}(K) .
$$

Here $\boldsymbol{a}=\boldsymbol{c}^{-1}$ and $\mathcal{P}_{0}^{k+1}(K)$ is the set of polynomials in $\mathcal{P}^{k+1}(K)$ with zero mean.

We are now ready to state the result.

Theorem 2.4. Assume that $\Omega$ is such that the elliptic regularity result (2.12) holds with $r=0$. Then, for any method of the form (1.2) satisfying the inclusions (2.6) for $k \geq 1$, we have that

$$
\begin{array}{r}
\left\|u-u_{h}^{\star}\right\|_{L^{2}\left(\Omega_{h}\right)} \leq C h\left(\left\|\boldsymbol{q}-\boldsymbol{\Pi}_{k}^{\mathrm{RT}} \boldsymbol{q}\right\|_{L^{2}(\Omega)}+h\left\|f-\mathrm{P}^{k} f\right\|_{L^{2}\left(\Omega_{h}\right)}\right. \\
\left.+\left\|\nabla\left(\mathrm{P}^{k+1} u-u\right)\right\|_{\boldsymbol{L}^{2}\left(\Omega_{h}\right)}+\Phi_{k}\right),
\end{array}
$$

where

$$
\Phi_{k}:=\Theta_{k}+\| P_{\partial}^{k+1}\left(\widehat{\boldsymbol{q}}_{h}-\boldsymbol{q}_{h}\right) \boldsymbol{|}_{L^{2}\left(\mathscr{E}_{h} ; h\right)} .
$$

Note that for the RT method, $\Phi_{k}=0$ and we recover the corresponding result for that method; see [16, 17] and [14].

\section{EXAmples of SUPERCONVERGENT DG METHods}

In all the examples considered here, we take DG methods whose spaces are given by (1.3). As a consequence, we immediately have that

$$
\frac{1}{2} \Phi_{k}=\Theta_{k}=\left(\sum_{K \in \Omega_{h}} h_{K}\left\|\left(\widehat{\boldsymbol{q}}_{h}-\boldsymbol{q}_{h}\right) \cdot \boldsymbol{n}\right\|_{L^{2}(\partial K)}^{2}\right)^{1 / 2} .
$$

We are going to show for some DG methods and some LDG-H methods that we have

$$
\Phi_{k} \leq C\left(|u|_{H^{k+1}\left(\Omega_{h}\right)}+|\boldsymbol{q}|_{\boldsymbol{H}^{k+1}\left(\Omega_{h}\right)}+|f|_{H^{k}\left(\Omega_{h}\right)}\right) h^{k+1} .
$$

By our main results, this implies that, for $k \geq 0$,

$$
\begin{aligned}
\left\|\boldsymbol{q}-\boldsymbol{q}_{h}\right\|_{L^{2}(\Omega)} \leq C\left(|u|_{H^{k+1}\left(\Omega_{h}\right)}+|\boldsymbol{q}|_{\boldsymbol{H}^{k+1}\left(\Omega_{h}\right)}+|f|_{H^{k}\left(\Omega_{h}\right)}\right) h^{k+1}, \\
\left\|\boldsymbol{q}-\boldsymbol{q}_{h}^{\star}\right\|_{L^{2}(\Omega)} \leq C\left(|u|_{H^{k+1}\left(\Omega_{h}\right)}+|\boldsymbol{q}|_{\boldsymbol{H}^{k+1}\left(\Omega_{h}\right)}+|f|_{H^{k}\left(\Omega_{h}\right)}\right) h^{k+1},
\end{aligned}
$$

and for $k \geq 1$,

$$
\left\|u-u_{h}^{\star}\right\|_{L^{2}(\Omega)} \leq C\left(|u|_{H^{k+1}\left(\Omega_{h}\right)}+|u|_{H^{k+2}\left(\Omega_{h}\right)}+|\boldsymbol{q}|_{\boldsymbol{H}^{k+1}\left(\Omega_{h}\right)}+|f|_{H^{k}\left(\Omega_{h}\right)}\right) h^{k+2} .
$$


3.1. DG methods. We begin by considering the methods studied in [8]. Those are DG methods whose numerical traces on the interior faces are given by (1.4). On boundary faces, we take

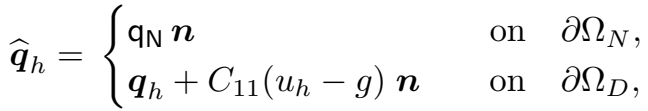

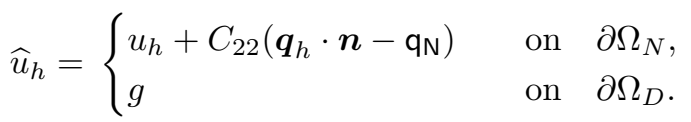

The result will be stated in terms of the quantities $\bar{C}_{i i}=\left.\max _{e \in \mathscr{E}_{h}} C_{i i}\right|_{e}$ and $\underline{C}_{i i}=\left.\min _{e \in \mathscr{E}_{h}} C_{i i}\right|_{e}$, for $i=1,2$. Also, we write $a_{h} \sim b_{h}$, for positive quantities $a_{h}$ and $b_{h}$ depending on the triangulation $\Omega_{h}$, when there is an arbitrary but fixed constant $c$ such that $b_{h} / c \leq a_{h} \leq c b_{h}$.

Proposition 3.1. We have

$$
\Phi_{k} \leq C\left(|u|_{H^{k+1}\left(\Omega_{h}\right)}+|\boldsymbol{q}|_{\boldsymbol{H}^{k+1}\left(\Omega_{h}\right)}+|f|_{H^{k}\left(\Omega_{h}\right)}\right) h^{k+1},
$$

provided

$$
\text { (i) } \quad\left|C_{12}\right| \leq \mathrm{C},
$$$$
\text { (ii) } \bar{C}_{22} \sim \underline{C}_{22} \sim \frac{1}{\bar{C}_{11}} \sim \frac{1}{\underline{C}_{11}},
$$$$
\text { (iii) } \quad \bar{C}_{11} \leq \mathrm{C},
$$

for some constant $\mathrm{C}$.

Note that, in particular, we can take $C_{11}=1 / C_{22}$ constant on $\mathscr{E}_{h}$. If such a constant is uniformly bounded, the DG method is optimally convergent in $\boldsymbol{q}_{h}$. This result improves the error estimates in [8], which predicted an order of $k+1 / 2$ if such a constant is independent of $h$. Of course, the DG methods under consideration are difficult to implement given that $C_{22}>0$. However, this is not the case for the LDG-H methods we consider next.

3.2. The LDG-H method. For the LDG-H method (see [10]), we have

$$
\widehat{\boldsymbol{q}}_{h}=\boldsymbol{q}_{h}+\tau\left(u_{h}-\widehat{u}_{h}\right) \boldsymbol{n},
$$

where $\tau$ is a nonnegative, piecewise constant, double-valued function on the interior faces of the triangulation and single-valued on boundary faces. We require $\left.\tau\right|_{\partial K} \not \equiv 0$ for each element $K$. The function $\widehat{u}_{h}$ is an unknown on interior faces and on $\partial \Omega_{N}$ and is equal to $\mathrm{P}_{\partial}^{k} g$ on $\partial \Omega_{D}$. We state our result in terms of some quantities we define next. For each $K \in \Omega_{h}$, let $e_{K}^{\tau}$ be an edge in which $\left.\tau\right|_{\partial K}$ is a maximum. Let $\bar{\tau}_{K}$ be the maximum value of $\left.\tau\right|_{\partial K \backslash e_{K}^{\tau}}$. Then we set $\bar{\tau}:=\max _{K \in \Omega_{h}} \bar{\tau}_{K}$. We also define $\underline{\tau}=\left.\min _{K \in \Omega_{h}} \tau\right|_{e_{K}^{\tau}}$.

Proposition 3.2. We have that

$$
\Phi_{k} \leq C\left(|u|_{H^{k+1}\left(\Omega_{h}\right)}+|\boldsymbol{q}|_{\boldsymbol{H}^{k+1}\left(\Omega_{h}\right)}+|f|_{H^{k}\left(\Omega_{h}\right)}\right) h^{k+1},
$$

provided

$$
\begin{array}{ll}
\text { (i) } & \bar{\tau} \leq \mathrm{C}_{1}, \\
\text { (ii) } & \frac{\bar{\tau}}{\underline{\tau}} \leq \mathrm{C}_{2},
\end{array}
$$

for some constants $\mathrm{C}_{1}$ and $\mathrm{C}_{2}$. 
The SF-H method [9] is a particular LDG-H method obtained by taking $\tau=0$ on $\partial K \backslash e_{K}^{\tau}$ and $\tau>0$ on $e_{K}^{\tau}$. With this choice, we get $\bar{\tau} \equiv 0$ and $\underline{\tau}>0$, and, as a consequence, Proposition 3.2 holds with $\mathrm{C}_{1}=\mathrm{C}_{2}=0$. In fact, in 9], it was directly shown that

$$
\left\|\left(\widehat{\boldsymbol{q}}_{h}-\boldsymbol{q}_{h}\right) \cdot \boldsymbol{n}\right\|_{L^{2}(\partial K)} \leq C h_{K}^{k+1 / 2}|f|_{H^{k}(K)},
$$

where $C$ is independent of $\left.\tau\right|_{\partial K}$, which immediately implies that

$$
\Phi_{k} \leq C h^{k+1}|f|_{H^{k}\left(\Omega_{h}\right)},
$$

independently of the values of $\tau$, provided $f \in H^{k}\left(\Omega_{h}\right)$.

\section{Proofs}

To prove the theorems of the previous section, we proceed in several steps. We begin by introducing the main tools of the analysis, namely, the error equations and some suitably defined projections.

As is traditional in the analysis of finite element methods, we begin by obtaining the error equations. From the definition of the exact solution (1.1) and the general form of the methods under consideration (1.2), we easily obtain the following error equations:

$$
\begin{aligned}
& \left(\boldsymbol{c}\left(\boldsymbol{q}-\boldsymbol{q}_{h}\right), \boldsymbol{v}\right)_{\Omega_{h}}-\left(u-u_{h}, \nabla \cdot \boldsymbol{v}\right)_{\Omega_{h}}+\left\langle u-\widehat{u}_{h}, \boldsymbol{v} \cdot \boldsymbol{n}\right\rangle_{\partial \Omega_{h} \backslash \partial \Omega_{D}}=0, \\
& -\left(\boldsymbol{q}-\boldsymbol{q}_{h}, \nabla \omega\right)_{\Omega_{h}}+\left\langle\left(\boldsymbol{q}-\widehat{\boldsymbol{q}}_{h}\right) \cdot \boldsymbol{n}, \omega\right\rangle_{\partial \Omega_{h} \backslash \partial \Omega_{N}}=0,
\end{aligned}
$$

for all $(\boldsymbol{v}, \omega) \in \mathbf{V}_{h} \times \mathbf{W}_{h}$.

4.1. Some properties of $\boldsymbol{q}_{h}^{\star}$. To obtain error estimates for $\boldsymbol{q}_{h}^{\star}$, we begin by gathering properties of the function $\boldsymbol{q}_{h}^{\star}-\boldsymbol{\Pi}_{k}^{\mathrm{RT}} \boldsymbol{q}$ in the following lemma.

Lemma 4.1. Assume that the inclusion property (2.6b) for the space $\mathrm{W}_{h}$ holds. Assume also that the property (1.2d) of single-valuedness of the numerical trace $\widehat{\boldsymbol{q}}_{h}$ holds. Then

(i) $\left(\boldsymbol{q}_{h}^{\star}-\boldsymbol{\Pi}_{k}^{\mathrm{RT}} \boldsymbol{q}\right) \cdot \boldsymbol{n}=0$ on $\partial \Omega_{N}$,

(ii) $\boldsymbol{q}_{h}^{\star}-\boldsymbol{\Pi}_{k}^{\mathrm{RT}} \boldsymbol{q} \in H(\operatorname{div}, \Omega)$,

(iii) $\nabla \cdot\left(\boldsymbol{q}_{h}^{\star}-\boldsymbol{\Pi}_{k}^{\mathrm{RT}} \boldsymbol{q}\right)=0$ in $\Omega$,

(iv) $\boldsymbol{q}_{h}^{\star}-\boldsymbol{\Pi}_{k}^{\mathrm{RT}} \boldsymbol{q} \in \mathbf{V}_{h}^{k}$.

Proof. Let us prove property (i). We have that, on any face $e$ lying on $\partial \Omega_{N}$,

$$
\begin{aligned}
\boldsymbol{q}_{h}^{\star} \cdot \boldsymbol{n} & \left.=\mathrm{P}_{\partial}^{k}\left(\widehat{\boldsymbol{q}}_{h} \cdot \boldsymbol{n}\right) \text { by property (2.9a) }\right) \\
& =\mathrm{P}_{\partial}^{k}\left(\boldsymbol{q}_{\mathrm{N}}\right) \quad \text { by the Neumann boundary condition (1.2f) }, \\
& =\Pi_{k}^{\mathrm{RT}} \boldsymbol{q} \cdot \boldsymbol{n} \quad \text { by the definition of the projection } \boldsymbol{\Pi}_{k}^{\mathrm{RT}}(2.7 \mathrm{a}) .
\end{aligned}
$$

Property (ii) follows by the definition of the projection $\boldsymbol{\Pi}_{k}^{\mathrm{RT}}$, the definition of $\boldsymbol{q}_{h}^{\star}$, and by the fact that the normal component of the numerical trace for the flux is single-valued, (1.2d).

Next, let us prove property (iii). By the inclusion property (2.6b), we can rewrite the equation (1.2b) as follows:

$$
\left(\nabla \cdot \boldsymbol{q}_{h}^{\star}, w\right)_{\Omega_{h}}=(f, w)_{\Omega_{h}} \quad \text { for all } w \in \mathbf{W}_{h}^{k} .
$$


As a consequence, we have that

$$
\left(\nabla \cdot\left(\boldsymbol{q}_{h}^{\star}-\boldsymbol{\Pi}_{k}^{\mathrm{RT}} \boldsymbol{q}\right), w\right)_{\Omega_{h}}=0 \quad \text { for all } w \in \mathbf{W}_{h}^{k},
$$

and since $\nabla \cdot\left(\boldsymbol{q}_{h}^{\star}-\mathbf{\Pi}_{k}^{\mathrm{RT}} \boldsymbol{q}\right) \in \mathrm{W}_{h}^{k}$, (iii) follows.

Property (iv) is a direct consequence of property (iii) and the fact that, for each simplex $K \in \Omega_{h}, \boldsymbol{q}_{h}^{\star}-\left.\boldsymbol{\Pi}_{k}^{\mathrm{RT}} \boldsymbol{q}\right|_{K} \in \mathcal{P}^{k}(K)+\boldsymbol{x} \mathcal{P}^{k}(K)$, which follows from the definitions of the projection $\boldsymbol{\Pi}_{k}^{\mathrm{RT}}$ (2.7) and the function $\boldsymbol{q}_{h}^{\star}$ (2.9). This completes the proof.

We are now ready to obtain an estimate of $\boldsymbol{q}_{h}^{\star}-\boldsymbol{\Pi}_{k}^{\mathrm{RT}} \boldsymbol{q}_{h}$.

Lemma 4.2. We have

$$
\left\|\boldsymbol{q}_{h}^{\star}-\boldsymbol{\Pi}_{k}^{\mathrm{RT}} \boldsymbol{q}_{h}\right\|_{L^{2}(K)} \leq C h_{K}^{1 / 2}\left\|\mathrm{P}_{\partial}^{k}\left(\widehat{\boldsymbol{q}}_{h}-\boldsymbol{q}_{h}\right) \cdot \boldsymbol{n}\right\|_{L^{2}(\partial K)} .
$$

Proof. If we set $\boldsymbol{\delta}:=\boldsymbol{q}_{h}^{\star}-\boldsymbol{\Pi}_{k}^{\mathrm{RT}} \boldsymbol{q}_{h}$, then on the simplex $K \in \Omega_{h}$, the function $\boldsymbol{\delta}$ is the element of $\mathcal{P}^{k}(K)+\boldsymbol{x} \mathcal{P}^{k}(K)$ satisfying the equations

$$
\begin{aligned}
& (\boldsymbol{\delta}, \boldsymbol{v})_{K}=0, \quad \forall \boldsymbol{v} \in \mathcal{P}^{k-1}(K), \text { if } k \geq 1, \\
& \langle\boldsymbol{\delta} \cdot \boldsymbol{n}, \omega\rangle_{e}=\left\langle\mathrm{P}_{\partial}^{k}\left(\left(\widehat{\boldsymbol{q}}_{h}-\boldsymbol{q}_{h}\right) \cdot \boldsymbol{n}\right), \omega\right\rangle_{e} \quad \forall \omega \in \mathcal{P}^{k}(e) \text {, for all faces of } K \text {. }
\end{aligned}
$$

The result now easily follows; see [6]. This completes the proof.

4.2. Optimal convergence of $\boldsymbol{q}_{h}$ and $\boldsymbol{q}_{h}^{\star}$. Here we prove Theorems 2.1 and 2.2.

Let us begin by proving Theorem 2.2. The fact that $\boldsymbol{q}_{h}^{\star} \in H(\operatorname{div}, \Omega)$ follows from (ii) of Lemma 4.1 and the fact that $\boldsymbol{\Pi}_{k}^{\mathrm{RT}} \boldsymbol{q}$ is also in $H(\operatorname{div}, \Omega)$. The estimate of its divergence follows from (iii) of Lemma 4.1. Indeed, by such a property,

$$
\begin{aligned}
\left\|\nabla \cdot\left(\boldsymbol{q}-\boldsymbol{q}_{h}^{\star}\right)\right\|_{L^{2}(\Omega)} & =\left\|\nabla \cdot\left(\boldsymbol{q}-\boldsymbol{\Pi}_{k}^{\mathrm{RT}} \boldsymbol{q}\right)\right\|_{L^{2}(\Omega)} \\
& =\left\|f-\mathrm{P}^{k} f\right\|_{L^{2}\left(\Omega_{h}\right)},
\end{aligned}
$$

by a well-known property of the projection $\boldsymbol{\Pi}_{k}^{\mathrm{RT}}$; see, for example, $[6$. To prove the remaining inequality, we proceed as follows. We use the following notation:

$$
\|\boldsymbol{v}\|_{L^{2}(\Omega ; \boldsymbol{c})}:=(\boldsymbol{c} \boldsymbol{v}, \boldsymbol{v})_{\Omega}^{1 / 2} .
$$

By the first equation defining the method (1.2a),

$$
\left(\boldsymbol{c} \boldsymbol{q}_{h}, \boldsymbol{v}\right)_{\Omega_{h}}=\left(u_{h}, \nabla \cdot \boldsymbol{v}\right)_{\Omega_{h}}-\left\langle\widehat{u}_{h}, \boldsymbol{v} \cdot \boldsymbol{n}\right\rangle_{\partial \Omega_{h}} \quad \text { for all } \boldsymbol{v} \in \mathbf{V}_{h} \cap H(\operatorname{div}, \Omega) .
$$

As a consequence, since the numerical trace $\widehat{u}_{h}$ is single-valued (1.2c), we obtain that

$$
\left(\boldsymbol{c} \boldsymbol{q}_{h}, \boldsymbol{v}\right)_{\Omega_{h}}=\left(u_{h}, \nabla \cdot \boldsymbol{v}\right)_{\Omega_{h}}-\left\langle\widehat{u}_{h}, \boldsymbol{v} \cdot \boldsymbol{n}\right\rangle_{\partial \Omega} \quad \text { for all } \boldsymbol{v} \in \mathbf{V}_{h} \cap H(\operatorname{div}, \Omega) .
$$

This implies that, for all $\boldsymbol{v} \in \mathbf{V}_{h} \cap H(\operatorname{div}, \Omega)$,

$$
\left(\boldsymbol{c}\left(\boldsymbol{q}-\boldsymbol{q}_{h}\right), \boldsymbol{v}\right)_{\Omega_{h}}=\left(u-u_{h}, \nabla \cdot \boldsymbol{v}\right)_{\Omega_{h}}-\left\langle u-\widehat{u}_{h}, \boldsymbol{v} \cdot \boldsymbol{n}\right\rangle_{\partial \Omega_{N}},
$$

by the Dirichlet boundary condition on the exact solution (1.1c) and on the approximate solution (1.2e).

We claim that we can take $\boldsymbol{v}=\boldsymbol{\Pi}_{k}^{\mathrm{RT}} \boldsymbol{q}-\boldsymbol{q}_{h}^{\star}$ in the previous equation. Indeed, by property (iv) of Lemma4.1 the function $\boldsymbol{\Pi}_{k}^{\mathrm{RT}} \boldsymbol{q}-\boldsymbol{q}_{h}^{\star}$ belongs to $\mathbf{V}_{h}^{k}$, and by property (ii) of Lemma 4.1, it also belongs to $H(\operatorname{div}, \Omega)$. Hence, it belongs to $\mathbf{V}_{h}^{k} \cap H(\operatorname{div}, \Omega)$ 
and so to $\mathbf{V}_{h} \cap H(\operatorname{div}, \Omega)$, by the inclusion property (2.6a). As a consequence, we get that

$$
\left(\boldsymbol{c}\left(\boldsymbol{q}-\boldsymbol{q}_{h}\right), \boldsymbol{\Pi}_{k}^{\mathrm{RT}} \boldsymbol{q}-\boldsymbol{q}_{h}^{\star}\right)_{\Omega_{h}}=0,
$$

by properties (i) and (iii) of Lemma 4.1.

This implies that

$$
\begin{aligned}
\left\|\boldsymbol{\Pi}_{k}^{\mathrm{RT}} \boldsymbol{q}-\boldsymbol{q}_{h}^{\star}\right\|_{L^{2}\left(\Omega_{h} ; \boldsymbol{c}\right)}^{2}=\left(\boldsymbol{c}\left(\boldsymbol{\Pi}_{k}^{\mathrm{RT}} \boldsymbol{q}-\boldsymbol{q}\right), \boldsymbol{\Pi}_{k}^{\mathrm{RT}} \boldsymbol{q}-\boldsymbol{q}_{h}^{\star}\right)_{\Omega_{h}} \\
-\left(\boldsymbol{c}\left(\boldsymbol{q}_{h}^{\star}-\boldsymbol{q}_{h}\right), \boldsymbol{\Pi}_{k}^{\mathrm{RT}} \boldsymbol{q}-\boldsymbol{q}_{h}^{\star}\right)_{\Omega_{h}},
\end{aligned}
$$

and so,

$$
\begin{aligned}
\left\|\boldsymbol{\Pi}_{k}^{\mathrm{RT}} \boldsymbol{q}-\boldsymbol{q}_{h}^{\star}\right\|_{L^{2}\left(\Omega_{h} ; \boldsymbol{c}\right) \leq} & \left\|\boldsymbol{\Pi}_{k}^{\mathrm{RT}} \boldsymbol{q}-\boldsymbol{q}\right\|_{L^{2}\left(\Omega_{h} ; \boldsymbol{c}\right)}+\left\|\boldsymbol{q}_{h}^{\star}-\boldsymbol{q}_{h}\right\|_{L^{2}\left(\Omega_{h} ; \boldsymbol{c}\right)} \\
\leq \| & \left\|\boldsymbol{\Pi}_{k}^{\mathrm{RT}} \boldsymbol{q}-\boldsymbol{q}\right\|_{L^{2}\left(\Omega_{h} ; \boldsymbol{c}\right)}+\left\|\boldsymbol{q}_{h}^{\star}-\boldsymbol{\Pi}_{k}^{\mathrm{RT}} \boldsymbol{q}_{h}\right\|_{L^{2}\left(\Omega_{h} ; \boldsymbol{c}\right)} \\
& +\left\|\boldsymbol{\Pi}_{k}^{\mathrm{RT}} \boldsymbol{q}_{h}-\boldsymbol{q}_{h}\right\|_{L^{2}\left(\Omega_{h} ; \boldsymbol{c}\right)} \\
\leq \| & \left\|\boldsymbol{\Pi}_{k}^{\mathrm{RT}} \boldsymbol{q}-\boldsymbol{q}\right\|_{L^{2}\left(\Omega_{h} ; \boldsymbol{c}\right)}+C\left\|\mathrm{P}_{\partial}^{k}\left(\widehat{\boldsymbol{q}}_{h}-\boldsymbol{q}_{h}\right)\right\|_{L^{2}\left(\mathscr{E}_{h} ; h\right)} \\
& +\left\|\boldsymbol{\Pi}_{k}^{\mathrm{RT}} \boldsymbol{q}_{h}-\boldsymbol{q}_{h}\right\|_{L^{2}\left(\Omega_{h} ; \boldsymbol{c}\right)}
\end{aligned}
$$

since, by Lemma 4.2 ,

$$
\left\|\boldsymbol{q}_{h}^{\star}-\boldsymbol{\Pi}_{k}^{\mathrm{RT}} \boldsymbol{q}_{h}\right\|_{L^{2}(K)} \leq C h_{K}^{1 / 2}\left\|\mathrm{P}_{\partial}^{k}\left(\widehat{\boldsymbol{q}}_{h}-\boldsymbol{q}_{h}\right) \cdot \boldsymbol{n}\right\|_{L^{2}(\partial K)} .
$$

This implies that

$$
\left\|\boldsymbol{q}-\boldsymbol{q}_{h}^{\star}\right\|_{L^{2}\left(\Omega_{h}\right)} \leq C\left(\left\|\boldsymbol{\Pi}_{k}^{\mathrm{RT}} \boldsymbol{q}-\boldsymbol{q}\right\|_{L^{2}\left(\Omega_{h}\right)}+\Theta_{k}\right),
$$

by the triangle inequality and the definition of $\Theta_{k}$. This completes the proof of Theorem 2.2.

Let us now prove Theorem 2.1. Since

$\left\|\boldsymbol{q}-\boldsymbol{q}_{h}\right\|_{L^{2}\left(\Omega_{h}\right)} \leq\left\|\boldsymbol{q}-\boldsymbol{q}_{h}^{\star}\right\|_{L^{2}\left(\Omega_{h}\right)}+\left\|\boldsymbol{q}_{h}^{\star}-\boldsymbol{\Pi}_{k}^{\mathrm{RT}} \boldsymbol{q}_{h}\right\|_{L^{2}\left(\Omega_{h}\right)}+\left\|\boldsymbol{\Pi}_{k}^{\mathrm{RT}} \boldsymbol{q}_{h}-\boldsymbol{q}_{h}\right\|_{L^{2}\left(\Omega_{h}\right)}$, we only have to use Lemma 4.2 and the first inequality of Theorem 2.1 to obtain our estimate. This completes the proof of Theorem 2.1 .

4.3. Superconvergence of $\mathrm{P}^{k-1} u_{h}$ in $\Omega$. Next we prove the first superconvergence estimate of Theorem 2.3 .

We begin by noting that

$$
\begin{aligned}
\Xi_{h} & :=\left(\mathrm{P}^{k-1}\left(u-u_{h}\right), \theta\right)_{\Omega_{h}} \\
& =\left(\mathrm{P}^{k-1}\left(u-u_{h}\right), \nabla \cdot \boldsymbol{\psi}\right)_{\Omega_{h}} \\
& =\left(u-u_{h}, \nabla \cdot \boldsymbol{\Pi}_{k-1}^{\mathrm{RT}} \boldsymbol{\psi}\right)_{\Omega_{h}} .
\end{aligned}
$$

By the inclusion (2.6a), we can take $\boldsymbol{v}=\boldsymbol{\Pi}_{k-1}^{\mathrm{RT}} \boldsymbol{\psi}$ in the error equation (4.16a) and obtain

$$
\begin{aligned}
\Xi_{h} & =\left(\boldsymbol{c}\left(\boldsymbol{q}-\boldsymbol{q}_{h}\right), \boldsymbol{\Pi}_{k-1}^{\mathrm{RT}} \boldsymbol{\psi}\right)_{\Omega_{h}} \\
& =\left(\boldsymbol{c}\left(\boldsymbol{q}-\boldsymbol{q}_{h}\right), \boldsymbol{\Pi}_{k-1}^{\mathrm{RT}} \boldsymbol{\psi}-\boldsymbol{\psi}\right)_{\Omega_{h}}+\left(\boldsymbol{c}\left(\boldsymbol{q}-\boldsymbol{q}_{h}\right), \boldsymbol{\psi}\right)_{\Omega_{h}} \\
& =\left(\boldsymbol{c}\left(\boldsymbol{q}-\boldsymbol{q}_{h}\right), \boldsymbol{\Pi}_{k-1}^{\mathrm{RT}} \boldsymbol{\psi}-\boldsymbol{\psi}\right)_{\Omega_{h}}-\left(\boldsymbol{q}-\boldsymbol{q}_{h}, \nabla \phi\right)_{\Omega_{h}}
\end{aligned}
$$

by the adjoint equation (2.11a). Hence

$$
\Xi_{h}=\sum_{i=1}^{4} T_{i},
$$


where

$$
\begin{aligned}
T_{1} & :=\left(\boldsymbol{c}\left(\boldsymbol{q}-\boldsymbol{q}_{h}\right), \boldsymbol{\Pi}_{k-1}^{\mathrm{RT}} \boldsymbol{\psi}-\boldsymbol{\psi}\right)_{\Omega_{h}}, \\
T_{2} & :=-\left(\boldsymbol{q}-\boldsymbol{\Pi}_{k}^{\mathrm{RT}} \boldsymbol{q}, \nabla \phi\right)_{\Omega_{h}}, \\
T_{3} & :=-\left(\boldsymbol{\Pi}_{k}^{\mathrm{RT}} \boldsymbol{q}-\boldsymbol{q}_{h}^{\star}, \nabla \phi\right)_{\Omega_{h}}, \\
T_{4} & :=-\left(\boldsymbol{q}_{h}^{\star}-\boldsymbol{q}_{h}, \nabla \phi\right)_{\Omega_{h}} .
\end{aligned}
$$

Let us estimate each of these terms. Since $k \geq 1$, we have that

$$
\left|T_{1}\right| \leq C h\left\|\boldsymbol{q}-\boldsymbol{q}_{h}\right\|_{L^{2}\left(\Omega_{h}\right)}\|\boldsymbol{\psi}\|_{H^{1}\left(\Omega_{h}\right)} .
$$

By the definition of the Raviart-Thomas projection, (2.7b),

$$
T_{2}=-\left(\boldsymbol{q}-\boldsymbol{\Pi}_{k}^{\mathrm{RT}} \boldsymbol{q}, \nabla \phi-\mathrm{P}^{0} \nabla \phi\right)_{\Omega_{h}},
$$

we obtain, since $k \geq 1$,

$$
\left|T_{2}\right| \leq C h\left\|\boldsymbol{q}-\boldsymbol{\Pi}_{k}^{\mathrm{RT}} \boldsymbol{q}\right\|_{L^{2}\left(\Omega_{h}\right)}\|\phi\|_{H^{2}\left(\Omega_{h}\right)} .
$$

By properties (i), (ii) and (iii) of Lemma 4.1.

$$
T_{3}=-\left\langle\left(\boldsymbol{\Pi}_{k}^{\mathrm{RT}} \boldsymbol{q}-\boldsymbol{q}_{h}^{\star}\right) \cdot \boldsymbol{n}, \phi\right\rangle_{\partial \Omega_{D}}=0,
$$

by the adjoint equation (2.11c). Finally, by the definition of $\boldsymbol{q}_{h}^{\star},(2.9 \mathrm{~b})$,

$$
T_{4}=-\left(\boldsymbol{q}_{h}^{\star}-\boldsymbol{q}_{h}, \nabla \phi-\mathrm{P}^{0} \nabla \phi\right)_{\Omega_{h}}
$$

and so

$$
\left|T_{4}\right| \leq C h\left\|\boldsymbol{q}_{h}^{\star}-\boldsymbol{q}_{h}\right\|_{L^{2}\left(\Omega_{h}\right)}\|\phi\|_{H^{2}\left(\Omega_{h}\right)} .
$$

Finally, by Lemma 4.2,

$$
\left|T_{4}\right| \leq C h \Theta_{k}\|\phi\|_{H^{2}\left(\Omega_{h}\right)}
$$

This implies that

$$
\Xi_{h} \leq C h\left(\left\|\boldsymbol{q}-\boldsymbol{\Pi}_{k}^{\mathrm{RT}} \boldsymbol{q}\right\|_{L^{2}(\Omega)}+\Theta_{k}\right)\|\theta\|_{L^{2}\left(\Omega_{h}\right)}
$$

by the elliptic regularity estimate (2.12) with $r=0$. This completes the proof of the first estimate of Theorem 2.3 .

4.4. Superconvergence of $\mathrm{P}_{\partial}^{k} u_{h}$ on $\mathscr{E}_{h}$. Here, we prove the second estimate of Theorem 2.3. We begin by the following simple result.

Lemma 4.3. For any method of the form (1.2) satisfying the inclusion (2.6a) for the space $\mathbf{V}_{h}$, we have that

$$
h_{K}^{1 / 2}\left\|\mathrm{P}_{\partial}^{k}\left(u-\widehat{u}_{h}\right)\right\|_{L^{2}(e)} \leq C h\left\|\boldsymbol{q}-\boldsymbol{q}_{h}\right\|_{L^{2}(K)}+C\left\|\mathrm{P}^{k-1}\left(u-u_{h}\right)\right\|_{L^{2}(K)},
$$

for any face e of $\partial K$ and any simplex $K \in \Omega_{h}$.

Proof. Thanks to the inclusion property (2.6a), from the error equation (4.16a) we can deduce that

$$
\left\langle\mathrm{P}_{\partial}^{k}\left(u-\widehat{u}_{h}\right), \boldsymbol{v} \cdot n\right\rangle_{\partial K}=-\left(\boldsymbol{c}\left(\boldsymbol{q}-\boldsymbol{q}_{h}\right), \boldsymbol{v}\right)_{K}+\left(\mathrm{P}^{k-1}\left(u-u_{h}\right), \nabla \cdot \boldsymbol{v}\right)_{K} \quad \forall \boldsymbol{v} \in \mathfrak{P}^{k}(K) .
$$


If we now choose a $\boldsymbol{v} \in \mathcal{P}^{k}(K)$ such that

$$
\begin{aligned}
\boldsymbol{v} \cdot \boldsymbol{n} & =\mathrm{P}_{\partial}^{k}\left(u-\widehat{u}_{h}\right) & & \text { on } e, \\
\boldsymbol{v} \cdot \boldsymbol{n} & =0 & & \text { on } \partial K \backslash e, \\
(\boldsymbol{v}, \nabla w)_{K} & =0, & & \forall w \in \mathcal{P}^{k-1}(K), \\
\left(\boldsymbol{v}, \boldsymbol{v}^{\circ}\right)_{K} & =0, & & \forall \boldsymbol{v}^{\circ} \in \mathbf{\Phi}_{k}(K),
\end{aligned}
$$

where

$$
\boldsymbol{\Phi}_{k}(K)=\left\{\boldsymbol{v} \in \mathcal{P}^{k}(K): \nabla \cdot \boldsymbol{v}=0,\left.\boldsymbol{v} \cdot \boldsymbol{n}\right|_{\partial K}=0\right\},
$$

see [6], we readily obtain that

$$
\left\|\mathrm{P}_{\partial}^{k}\left(u-\widehat{u}_{h}\right)\right\|_{L^{2}(e)}^{2}=-\left(\boldsymbol{c}\left(\boldsymbol{q}-\boldsymbol{q}_{h}\right), \boldsymbol{v}\right)_{K}+\left(\mathrm{P}^{k-1}\left(u-u_{h}\right), \nabla \cdot \boldsymbol{v}\right)_{K} .
$$

The result now follows by noting that

$$
h_{K}\|\boldsymbol{v}\|_{H^{1}(K)}+\|\boldsymbol{v}\|_{L^{2}(K)} \leq C h_{K}^{1 / 2}\left\|\mathrm{P}_{\partial}^{k}\left(u-\widehat{u}_{h}\right)\right\|_{L^{2}(e)},
$$

which in turn follows from a scaling argument.

The second estimate of Theorem 2.3 follows from the above result, Theorem 2.1, and the first estimate of Theorem 2.3

4.5. Superconvergence of $u_{h}^{\star}$. It remains to prove Theorem 2.4. To do that, we follow 9. By the definition of $u_{h}^{\star}$, 2.13a), we have that

$$
\left\|u-u_{h}^{\star}\right\|_{L^{2}(K)} \leq \frac{1}{|K|}\left\|\int_{K}\left(u-u_{h}\right) d x\right\|_{L^{2}(K)}+\left\|\widetilde{u}-\widetilde{u}_{h}\right\|_{L^{2}(K)},
$$

where $\widetilde{u}$ stands for $u$ minus its average on $K$. Then

$$
\begin{aligned}
\left\|u-u_{h}^{\star}\right\|_{L^{2}(K)} & =\left\|\mathrm{P}^{0}\left(u-u_{h}\right)\right\|_{L^{2}(K)}+\left\|\widetilde{u}-\widetilde{u}_{h}\right\|_{L^{2}(K)} \\
& \leq\left\|\mathrm{P}^{k-1}\left(u-u_{h}\right)\right\|_{L^{2}(K)}+\left\|\widetilde{u}-\widetilde{u}_{h}\right\|_{L^{2}(K)},
\end{aligned}
$$

since $k-1 \geq 0$. This implies

$$
\left\|u-u_{h}^{\star}\right\|_{L^{2}\left(\Omega_{h}\right)} \leq C h\left(\left\|\boldsymbol{q}-\boldsymbol{\Pi}_{k}^{\mathrm{RT}} \boldsymbol{q}\right\|_{L^{2}(\Omega)}+\Theta_{k}\right)+\left\|\widetilde{u}-\widetilde{u}_{h}\right\|_{L^{2}\left(\Omega_{h}\right)} .
$$

It remains to estimate the second term of the right-hand side. Since, by Poincaré's inequality, we have

$$
\left\|\widetilde{u}-\widetilde{u}_{h}\right\|_{L^{2}(K)} \leq C h_{K}\left\|\nabla\left(\widetilde{u}-\widetilde{u}_{h}\right)\right\|_{L^{2}(K)},
$$

it is enough to estimate the error in the gradient. To do that, we note that, by the definition of $\widetilde{u}_{h},(2.13 \mathrm{~b})$, we have

$$
\left(\boldsymbol{a} \nabla\left(\widetilde{u}-\widetilde{u}_{h}\right), \nabla w\right)_{K}=-\left\langle w,\left(\boldsymbol{q}-\widehat{\boldsymbol{q}}_{h}\right) \cdot \boldsymbol{n}\right\rangle_{\partial K} \quad \forall w \in \mathcal{P}_{0}^{k+1}(K) .
$$


Then

$$
\begin{aligned}
\left\|\nabla\left(\mathrm{P}^{k+1} \widetilde{u}-\widetilde{u}_{h}\right)\right\|_{L^{2}(K ; \boldsymbol{a})}^{2}= & \left(\boldsymbol{a} \nabla\left(\widetilde{u}-\widetilde{u}_{h}\right), \nabla\left(\mathrm{P}^{k+1} \widetilde{u}-\widetilde{u}_{h}\right)\right)_{K} \\
& +\left(\boldsymbol{a} \nabla\left(\mathrm{P}^{k+1} \widetilde{u}-\widetilde{u}\right), \nabla\left(\mathrm{P}^{k+1} \widetilde{u}-\widetilde{u}_{h}\right)\right)_{K} \\
= & -\left\langle\mathrm{P}^{k+1} \widetilde{u}-\widetilde{u}_{h},\left(\boldsymbol{q}-\widehat{\boldsymbol{q}}_{h}\right) \cdot \boldsymbol{n}\right\rangle_{\partial K} \\
& +\left(\boldsymbol{a} \nabla\left(\mathrm{P}^{k+1} \widetilde{u}-\widetilde{u}\right), \nabla\left(\mathrm{P}^{k+1} \widetilde{u}-\widetilde{u}_{h}\right)\right)_{K}, \\
= & -\left\langle\mathrm{P}^{k+1} \widetilde{u}-\widetilde{u}_{h},\left(\boldsymbol{q}-\widehat{\boldsymbol{q}}_{h}\right) \cdot \boldsymbol{n}\right\rangle_{\partial K} \\
& +\left(\boldsymbol{a} \nabla\left(\mathrm{P}^{k+1} u-u\right), \nabla\left(\mathrm{P}^{k+1} \widetilde{u}-\widetilde{u}_{h}\right)\right)_{K} .
\end{aligned}
$$

Let us estimate the first term of the right-hand side. For any arbitrary $\omega \in$ $\mathcal{P}_{0}^{k+1}(K)$, we have

$$
\begin{aligned}
\left\langle\omega,\left(\boldsymbol{q}-\widehat{\boldsymbol{q}}_{h}\right) \cdot \boldsymbol{n}\right\rangle_{\partial K} & =\left\langle\omega,\left(\boldsymbol{q}-\boldsymbol{q}_{h}\right) \cdot \boldsymbol{n}\right\rangle_{\partial K}+\left\langle\omega,\left(\boldsymbol{q}_{h}-\widehat{\boldsymbol{q}}_{h}\right) \cdot \boldsymbol{n}\right\rangle_{\partial K} \\
& =\sum_{i=1}^{3} T_{i},
\end{aligned}
$$

where

$$
\begin{aligned}
& T_{1}=\left(\nabla \omega, \boldsymbol{q}-\boldsymbol{q}_{h}\right)_{K}, \\
& T_{2}=\left(\omega, \nabla \cdot\left(\boldsymbol{q}-\boldsymbol{q}_{h}\right)\right)_{K}, \\
& T_{3}=\left\langle\omega,\left(\boldsymbol{q}_{h}-\widehat{\boldsymbol{q}}_{h}\right) \cdot \boldsymbol{n}\right\rangle_{\partial K} .
\end{aligned}
$$

By using the Cauchy-Schwarz inequality, we get that

$$
T_{1} \leq\|\nabla \omega\|_{L^{2}(K ; \boldsymbol{a})}\left\|\boldsymbol{q}-\boldsymbol{q}_{h}\right\|_{L^{2}(K ; \boldsymbol{c})} .
$$

By using the definition of the Raviart-Thomas projection $\boldsymbol{\Pi}_{k}^{\mathrm{RT}}$, we get

$$
\begin{aligned}
T_{2} & =\left(\omega, f-\mathrm{P}^{k} f\right)_{K}+\left(\omega, \nabla \cdot\left(\boldsymbol{\Pi}_{k}^{\mathrm{RT}} \boldsymbol{q}-\boldsymbol{q}_{h}\right)\right)_{K} \\
& \leq\|\omega\|_{L^{2}(K)}\left(\left\|f-\mathrm{P}^{k} f\right\|_{L^{2}(K)}+C h_{K}^{-1}\left\|\boldsymbol{q}-\boldsymbol{q}_{h}\right\|_{L^{2}(K ; \boldsymbol{c})}\right) \\
& \leq C\|\nabla \omega\|_{L^{2}(K ; \boldsymbol{a})}\left(h_{K}\left\|f-\mathrm{P}^{k} f\right\|_{L^{2}(K)}+\left\|\boldsymbol{q}-\boldsymbol{q}_{h}\right\|_{L^{2}(K ; \boldsymbol{c})}\right),
\end{aligned}
$$

by Poincaré's inequality. Finally,

$$
T_{3} \leq\|\omega\|_{L^{2}(\partial K)}\left\|\mathrm{P}_{\partial}^{k+1}\left(\left(\boldsymbol{q}_{h}-\widehat{\boldsymbol{q}}_{h}\right) \cdot \boldsymbol{n}\right)\right\|_{L^{2}(\partial K)},
$$

and, after applying a simple inverse inequality, we get

$$
T_{3} \leq C\|\nabla \omega\|_{L^{2}(K ; \boldsymbol{a})} h_{K}^{1 / 2}\left\|\mathrm{P}_{\partial}^{k+1}\left(\left(\boldsymbol{q}_{h}-\widehat{\boldsymbol{q}}_{h}\right) \cdot \boldsymbol{n}\right)\right\|_{L^{2}(\partial K)} .
$$

As a consequence,

$$
\begin{aligned}
\left\langle\omega,\left(\boldsymbol{q}-\widehat{\boldsymbol{q}}_{h}\right) \cdot \boldsymbol{n}\right\rangle_{\partial K} \leq C\|\nabla \omega\|_{L^{2}(K ; \boldsymbol{a})} & \left(\left\|\boldsymbol{q}-\boldsymbol{q}_{h}\right\|_{L^{2}(K ; \boldsymbol{c})}\right. \\
& +h_{K}^{1 / 2}\left\|\mathrm{P}_{\partial}^{k+1}\left(\left(\boldsymbol{q}_{h}-\widehat{\boldsymbol{q}}_{h}\right) \cdot \boldsymbol{n}\right)\right\|_{L^{2}(\partial K)} \\
& \left.+h_{K}\left\|f-\mathrm{P}^{k} f\right\|_{L^{2}(K)}\right) .
\end{aligned}
$$

This implies that

$$
\begin{aligned}
& \left\|\nabla\left(\mathrm{P}^{k+1} \widetilde{u}-\widetilde{u}_{h}\right)\right\|_{L^{2}(K ; \boldsymbol{a})} \\
& \qquad C\left(\left\|\boldsymbol{q}-\boldsymbol{q}_{h}\right\|_{L^{2}(K ; \boldsymbol{c})}+h_{K}^{1 / 2}\left\|\mathrm{P}_{\partial}^{k+1}\left(\left(\boldsymbol{q}_{h}-\widehat{\boldsymbol{q}}_{h}\right) \cdot \boldsymbol{n}\right)\right\|_{L^{2}(\partial K)}\right. \\
& \left.\quad+h_{K}\left\|f-\mathrm{P}^{k} f\right\|_{L^{2}(K)}+\left\|\nabla\left(\mathrm{P}^{k+1} u-u\right)\right\|_{L^{2}(K)}\right)
\end{aligned}
$$


and, by Poincare's inequality,

$$
\begin{aligned}
\left\|\mathrm{P}^{k+1} \widetilde{u}-\widetilde{u}_{h}\right\|_{L^{2}\left(\Omega_{h} ; \boldsymbol{a}\right)} \leq C h & \left(\Theta_{k}+\left\|\left.\mathrm{P}_{\partial}^{k+1}\left(\widehat{\boldsymbol{q}}_{h}-\boldsymbol{q}_{h}\right)\right|_{L^{2}\left(\mathscr{E}_{h} ; h\right)}+\right\| \boldsymbol{q}-\boldsymbol{\Pi}_{k}^{\mathrm{RT}} \boldsymbol{q} \|_{\boldsymbol{L}^{2}(\Omega)}\right. \\
& \left.+h\left\|f-\mathrm{P}^{k} f\right\|_{L^{2}\left(\Omega_{h}\right)}+\left\|\nabla\left(\mathrm{P}^{k+1} u-u\right)\right\|_{\boldsymbol{L}^{2}\left(\Omega_{h}\right)}\right) .
\end{aligned}
$$

This completes the proof of Theorem 2.4

\section{NumericAl EXAMPLES}

In this section, we carry out numerical experiments to validate the theoretical convergence properties of the LDG-H method.

To do that, we use uniform meshes obtained by discretizing $\Omega=\left(-\frac{1}{2}, \frac{1}{2}\right) \times\left(-\frac{1}{2}, \frac{1}{2}\right)$ with squares of side $2^{-l}$ which are then divided into two triangles as indicated in Figure 1; the resulting mesh is denoted by "mesh $=l$ ". The test problem we

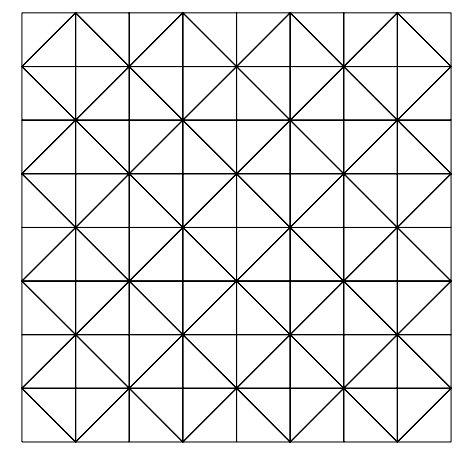

FIGURE 1. Example of a mesh with $h=1 / 2^{3}$.

consider here is obtained by taking $\partial \Omega_{N}=\emptyset, \boldsymbol{c}=\boldsymbol{I}$ and choosing $g$ and $f$ so that the exact solution is $u(x, y)=\cos (\pi x) \cos (\pi y)$ on the domain $\Omega$. It is the same as that considered in 9] where the SF-H method was studied.

In order to see the effect of $\tau$ on the orders of convergence of the errors, we begin by considering three cases: $\tau \equiv 1$ (Table 2), $\tau \equiv h$ (Table 3), $\tau=\frac{1}{h^{2}}$ on $e_{K}^{\tau}$ and $\tau=1$ on $\partial K \backslash e_{K}^{\tau}$ for all $K \in \Omega_{h}$ (Table 4). Since these three choices of $\tau$ satisfy conditions (i) and (ii) of Proposition 3.2, we expect to see the order of convergence predicted by Theorems 2.1] and 2.2. that is, $k+1$ for $\left\|\boldsymbol{q}-\boldsymbol{q}_{h}\right\|_{L^{2}(\Omega)}$ and $\left\|\boldsymbol{q}-\boldsymbol{q}_{h}^{\star}\right\|_{L^{2}(\Omega)}$, provided $k \geq 0$, and the orders of convergence predicted by Theorems 2.3 and 2.4 that is, $k+2$ for $\left\|P_{\partial} u-\widehat{u}_{h}\right\|_{L^{2}\left(\varepsilon_{h} ; h\right)}$ and $\left\|u-u_{h}^{\star}\right\|_{L^{2}(\Omega)}$, provided $k \geq 1$. We can see in Tables 2,3 and 4 that these orders of convergence are actually achieved in full agreement with the theory.

It is interesting to note that in all these experiments, the error of the postprocessed flux $\boldsymbol{q}_{h}^{\star}$ is actually smaller than that of the original flux $\boldsymbol{q}_{h}$. This shows that the postprocessed flux not only maintains the order of convergence of the original flux, but actually produces a slightly better approximation. These results are similar to those obtained in the framework of approximations for the Navier-Stokes equations in [13. 
It is also interesting to see that, unlike for the two other choices for the parameters $\tau$, for the second choice the order of convergence of the approximate potential is not $k+1$, but only $k$.

Finally, note that although Theorems 2.3 and 2.4 do not say anything about superconvergence when $k=0$, our numerical experiments show that it is not reasonable to expect it. Indeed, we see in Table 2 that for $\tau \equiv 1$, superconvergence does not occur for the quantities $\left\|P_{\partial} u-\widehat{u}_{h}\right\|_{L^{2}\left(\mathcal{E}_{h} ; h\right)}$ and $\left\|u-u_{h}^{\star}\right\|_{L^{2}(\Omega)}$, where instead of the definition (2.13b), we use

$$
u_{h}^{\star}=\widetilde{u}_{h}+\left.\frac{1}{d} \sum_{e \in \partial K} \widehat{u}_{h}\right|_{e}
$$

see [9]. The same thing happens, see Table 4, for $\left.\tau\right|_{e_{K}^{\tau}}=\frac{1}{h^{2}}$ and $\tau=1$ on $\partial K \backslash e_{K}^{\tau}$ for all $K \in \Omega_{h}$. However, see Table 3, if $\tau \equiv h$ we do observe superconvergence. To find out if this occurs because we are taking $\tau$ constant, we choose $\left.\tau\right|_{\partial K}$ to be $h$ on one edge of $\partial K, 2 h$ on the second edge, and $3 h$ on the last edge. The results, see Table 5, show that we no longer see superconvergence if $k=0$.

We end with an experiment devised to verify that the condition (i) of Proposition 3.2 is necessary. We choose $\tau \equiv \frac{1}{h}$ so that such a condition is not satisfied and see in Table 6 that the order of convergence of $\left\|\boldsymbol{q}-\boldsymbol{q}_{h}\right\|_{L^{2}(\Omega)}$ is not $k+1$ but only $k$. Note that for $k=0,\left\|\boldsymbol{q}-\boldsymbol{q}_{h}^{\star}\right\|_{L^{2}(\Omega)}$ converges with order one. However, if we take $\left.\tau\right|_{\partial K}$ to be $1 / h$ on one edge of $\partial K, 2 / h$ on the second edge, and $3 / h$ on the last edge, the convergence is lost.

\section{Concluding Remarks}

In this paper, we have used the RT projection and simplices to obtain Theorems 2.1 and 2.3. In a forthcoming paper, we consider general second-order elliptic equations and show how to use the BDM projection to obtain similar results.

\section{Appendix}

Here we are going to provide detailed proofs of Propositions 3.1 and 3.2, which contain the estimate of

$$
\Theta_{k}=\left(\sum_{K \in \Omega_{h}} h_{K}\left\|\left(\widehat{\boldsymbol{q}}_{h}-\boldsymbol{q}_{h}\right) \cdot \boldsymbol{n}\right\|_{L^{2}(\partial K)}^{2}\right)^{1 / 2}
$$

for the DG and LDG-H methods, respectively. To do that, we begin by obtaining an energy. We then particularize it to each of the two methods under consideration in order to deduce the estimate we seek.

7.1. The energy estimate. We begin by introducing two projections, denoted by $\Pi$ and $\mathbb{P}$, whose use is crucial in our analysis. To define them, for each simplex $K \in \Omega_{h}$, we have to single out a particular face, which we denote by $e_{K}^{\tau}$. The precise way in which we pick this face is not relevant for the study of the DG methods. When studying the LDG-H methods, we take $e_{K}^{\tau}$ to be the face of $K$ on which $\tau$ is a maximum on $\partial K$. 
TABLE 2. History of convergence for $\tau \equiv 1$.

\begin{tabular}{|c|c|c|c|c|c|c|c|c|c|c|c|}
\hline \multirow[b]{2}{*}{$k$} & \multirow{2}{*}{$\begin{array}{c}\text { mesh } \\
\ell\end{array}$} & \multicolumn{2}{|c|}{$\left\|u-u_{h}\right\|_{L^{2}(\Omega)}$} & \multicolumn{2}{|c|}{$\left\|\boldsymbol{q}-\boldsymbol{q}_{h}\right\|_{L^{2}(\Omega)}$} & \multicolumn{2}{|c|}{$\left\|\boldsymbol{q}-\boldsymbol{q}_{h}^{\star}\right\|_{L^{2}(\Omega)}$} & \multicolumn{2}{|c|}{$\left\|P_{\partial} u-\widehat{u}_{h}\right\|_{L^{2}\left(\varepsilon_{h} ; h\right)}$} & \multicolumn{2}{|c|}{$\left\|u-u_{h}^{\star}\right\|_{L^{2}(\Omega)}$} \\
\hline & & error & order & error & order & error & order & error & order & error & order \\
\hline \multirow{6}{*}{0} & 1 & $.62 \mathrm{e}-0$ & - & $.14 \mathrm{e}+1$ & - & $.97 \mathrm{e}-0$ & - & $.98 \mathrm{e}-1$ & - & $.16 \mathrm{e}-0$ & - \\
\hline & 2 & $.32 \mathrm{e}-0$ & 0.96 & $.68 \mathrm{e}-0$ & 1.04 & $.51 \mathrm{e}-0$ & 0.94 & $.92 \mathrm{e}-1$ & $.8 \mathrm{e}-1$ & $.67 \mathrm{e}-1$ & 1.23 \\
\hline & 3 & $.17 \mathrm{e}-0$ & 0.94 & $.34 \mathrm{e}-0$ & 0.99 & $.25 \mathrm{e}-0$ & 1.01 & $.43 \mathrm{e}-1$ & 1.10 & $.28 \mathrm{e}-1$ & 1.26 \\
\hline & 4 & $.84 \mathrm{e}-1$ & 0.97 & $.17 \mathrm{e}-0$ & 1.00 & $.13 \mathrm{e}-0$ & 1.00 & $.21 \mathrm{e}-1$ & 1.04 & $.12 \mathrm{e}-1$ & 1.16 \\
\hline & 5 & $.43 \mathrm{e}-1$ & 0.99 & $.86 \mathrm{e}-1$ & 1.00 & $.63 \mathrm{e}-1$ & 1.00 & $.10 \mathrm{e}-1$ & 1.02 & $.59 \mathrm{e}-2$ & 1.08 \\
\hline & 6 & $.21 \mathrm{e}-1$ & 0.99 & $.43 \mathrm{e}-1$ & 1.00 & $.31 \mathrm{e}-1$ & 1.00 & $.51 \mathrm{e}-2$ & 1.01 & $.28 \mathrm{e}-2$ & 1.04 \\
\hline \multirow{6}{*}{1} & 1 & $.14 \mathrm{e}-0$ & - & $.24 \mathrm{e}-0$ & - & $.18 \mathrm{e}-0$ & - & $.43 \mathrm{e}-1$ & - & $.35 \mathrm{e}-1$ & - \\
\hline & 2 & $.48 \mathrm{e}-1$ & 1.59 & $.10 \mathrm{e}-1$ & 1.26 & $.58 \mathrm{e}-1$ & 1.67 & $.46 \mathrm{e}-2$ & 3.23 & $.34 \mathrm{e}-2$ & 3.35 \\
\hline & 3 & $.12 \mathrm{e}-1$ & 1.95 & $.25 \mathrm{e}-1$ & 1.98 & $.14 \mathrm{e}-1$ & 1.99 & $.56 \mathrm{e}-3$ & 3.04 & $.40 \mathrm{e}-3$ & 3.08 \\
\hline & 4 & $.32 \mathrm{e}-2$ & 1.98 & $.64 \mathrm{e}-2$ & 2.00 & $.36 \mathrm{e}-2$ & 2.00 & $.68 \mathrm{e}-4$ & 3.03 & $.48 \mathrm{e}-4$ & 3.05 \\
\hline & 5 & $.80 \mathrm{e}-3$ & 1.99 & $.16 \mathrm{e}-2$ & 2.00 & $.90 \mathrm{e}-3$ & 2.00 & $.85 e-5$ & 3.02 & $.60 \mathrm{e}-5$ & 3.03 \\
\hline & 6 & $.20 \mathrm{e}-3$ & 2.00 & $.40 \mathrm{e}-3$ & 2.00 & $.23 \mathrm{e}-3$ & 2.00 & $.11 \mathrm{e}-5$ & 3.01 & $.74 \mathrm{e}-6$ & 3.01 \\
\hline \multirow{6}{*}{2} & 1 & $.41 \mathrm{e}-1$ & - & $.46 \mathrm{e}-1$ & - & $.38 \mathrm{e}-1$ & - & $.45 \mathrm{e}-2$ & - & $.75 \mathrm{e}-2$ & - \\
\hline & 2 & $.44 \mathrm{e}-2$ & 3.23 & $.61 \mathrm{e}-2$ & 2.91 & $.42 \mathrm{e}-2$ & 3.14 & $.30 \mathrm{e}-3$ & 3.88 & $.38 \mathrm{e}-3$ & 4.32 \\
\hline & 3 & $.58 \mathrm{e}-3$ & 2.93 & $.73 \mathrm{e}-3$ & 3.07 & $.52 \mathrm{e}-3$ & 3.02 & $.19 \mathrm{e}-4$ & 4.00 & $.24 \mathrm{e}-4$ & 3.98 \\
\hline & 4 & $.74 \mathrm{e}-4$ & 2.97 & $.89 \mathrm{e}-4$ & 3.03 & $.65 \mathrm{e}-4$ & 2.99 & $.12 \mathrm{e}-5$ & 4.02 & $.15 \mathrm{e}-5$ & 4.00 \\
\hline & 5 & $.93 \mathrm{e}-5$ & 2.99 & $.11 \mathrm{e}-4$ & 3.02 & $.82 \mathrm{e}-5$ & 3.00 & $.73 \mathrm{e}-7$ & 4.01 & $.93 \mathrm{e}-7$ & 4.00 \\
\hline & 6 & $.12 \mathrm{e}-5$ & 2.99 & $.14 \mathrm{e}-5$ & 3.01 & $.10 e-5$ & 3.00 & $.45 \mathrm{e}-8$ & 4.01 & $.58 \mathrm{e}-8$ & 4.00 \\
\hline
\end{tabular}

For any $\boldsymbol{\sigma} \in \boldsymbol{H}^{1}\left(\Omega_{h}\right)$, the function $\boldsymbol{\Pi} \boldsymbol{\sigma}$ is the element of $\mathbf{V}_{h}^{k}$ defined as follows. For each $K \in \Omega_{h}, \Pi \boldsymbol{\sigma}$ is the element of $\mathcal{P}^{k}(K)$ satisfying

$$
\begin{aligned}
(\boldsymbol{\Pi} \boldsymbol{\sigma}-\boldsymbol{\sigma}, \boldsymbol{v})_{K}=0 & \forall \boldsymbol{v} \in \mathcal{P}^{k-1}(K), \\
\langle(\boldsymbol{\Pi} \boldsymbol{\sigma}-\boldsymbol{\sigma}) \cdot \boldsymbol{n}, \omega\rangle_{e}=0 & \forall \omega \in \mathcal{P}^{k}(e) \text { and all faces } e \neq e_{K}^{\tau} .
\end{aligned}
$$

For any $\zeta \in H^{1}\left(\Omega_{h}\right)$, the function $\mathbb{P} \zeta$ is the element of $\mathbf{W}_{h}^{k}$ defined as follows. For each $K \in \Omega_{h}, \mathbb{P} \zeta$ is the element of $\mathcal{P}^{k}(K)$ satisfying

$$
\begin{array}{rlrl}
(\mathbb{P} \zeta-\zeta, \mathrm{w})_{K} & =0 & \forall \mathrm{w} \in \mathcal{P}^{k-1}(K), \\
\langle\mathbb{P} \zeta-\zeta, \omega\rangle_{e_{K}^{\tau}}=0 & \forall \omega \in \mathcal{P}^{k}\left(e_{K}^{\tau}\right) .
\end{array}
$$

These projections are well defined and have optimal approximation properties; see 9]. Using them, we can get the following result.

Lemma 7.1. For any method of the form (1.2) with the spaces as in (1.3), we have

$$
\left\|\boldsymbol{\Pi} \boldsymbol{q}-\boldsymbol{q}_{h}\right\|_{L^{2}\left(\Omega_{h} ; \boldsymbol{c}\right)}^{2}+\sum_{i=1}^{3} T_{i}=\left(\boldsymbol{c}(\boldsymbol{\Pi} \boldsymbol{q}-\boldsymbol{q}), \boldsymbol{\Pi} \boldsymbol{q}-\boldsymbol{q}_{h}\right)_{\Omega_{h}},
$$


TABLE 3. History of convergence for $\tau \equiv h$.

\begin{tabular}{|c|c|c|c|c|c|c|c|c|c|c|c|}
\hline \multirow[b]{2}{*}{$k$} & \multirow{2}{*}{$\begin{array}{c}\text { mesh } \\
\ell\end{array}$} & \multicolumn{2}{|c|}{$\left\|u-u_{h}\right\|_{L^{2}(\Omega)}$} & \multicolumn{2}{|c|}{$\left\|\boldsymbol{q}-\boldsymbol{q}_{h}\right\|_{L^{2}(\Omega)}$} & \multicolumn{2}{|c|}{$\left\|\boldsymbol{q}-\boldsymbol{q}_{h}^{\star}\right\|_{L^{2}(\Omega)}$} & \multicolumn{2}{|c|}{$\left\|P_{\partial} u-\widehat{u}_{h}\right\|_{L^{2}\left(\varepsilon_{h} ; h\right)}$} & \multicolumn{2}{|c|}{$\left\|u-u_{h}^{\star}\right\|_{L^{2}(\Omega)}$} \\
\hline & & error & order & error & order & error & order & error & order & error & order \\
\hline \multirow{6}{*}{0} & 1 & $.13 \mathrm{e}+1$ & - & $.14 \mathrm{e}+1$ & - & $.97401 \mathrm{e}-0$ & - & $.11 \mathrm{e}-0$ & - & $.15 \mathrm{e}-0$ & - \\
\hline & 2 & $.14 \mathrm{e}+1$ & $-.77 \mathrm{e}-1$ & $.65 \mathrm{e}-0$ & 1.12 & $.50657 \mathrm{e}-0$ & 0.94 & $.48 \mathrm{e}-1$ & 1.26 & $.45 \mathrm{e}-1$ & 1.73 \\
\hline & 3 & $.14 \mathrm{e}+1$ & $-.57 \mathrm{e}-1$ & $.33 \mathrm{e}-0$ & 0.99 & $.25224 \mathrm{e}-0$ & 1.00 & $.12 \mathrm{e}-1$ & 2.03 & $.12 \mathrm{e}-1$ & 1.94 \\
\hline & 4 & $.14 \mathrm{e}+1$ & $-.14 \mathrm{e}-1$ & $.16 \mathrm{e}-0$ & 1.00 & $.12597 \mathrm{e}-0$ & 1.00 & $.29 \mathrm{e}-2$ & 2.01 & $.30 \mathrm{e}-2$ & 1.98 \\
\hline & 5 & $.14 \mathrm{e}+1$ & $-.17 \mathrm{e}-2$ & $.82 \mathrm{e}-1$ & 1.00 & $.62964 \mathrm{e}-1$ & 1.00 & $.74 \mathrm{e}-3$ & 2.00 & $.74 \mathrm{e}-3$ & 1.99 \\
\hline & 6 & $.14 \mathrm{e}+1$ & $-.13 \mathrm{e}-3$ & $.41 \mathrm{e}-1$ & 1.00 & $.31479 \mathrm{e}-1$ & 1.00 & $.18 \mathrm{e}-3$ & 2.00 & $.19 \mathrm{e}-3$ & 1.99 \\
\hline \multirow{6}{*}{1} & 1 & $.24 \mathrm{e}-0$ & - & $.20 \mathrm{e}-0$ & - & $.16946 \mathrm{e}-0$ & - & $.32 \mathrm{e}-01$ & - & $.26 \mathrm{e}-1$ & - \\
\hline & 2 & $.18 \mathrm{e}-0$ & 0.37 & $.97 \mathrm{e}-1$ & 1.07 & $.55172 \mathrm{e}-1$ & 1.62 & $.36 \mathrm{e}-02$ & 3.18 & $.24 \mathrm{e}-2$ & 3.47 \\
\hline & 3 & $.94 \mathrm{e}-1$ & 0.96 & $.24 \mathrm{e}-1$ & 1.98 & $.13765 \mathrm{e}-1$ & 2.00 & $.43 \mathrm{e}-03$ & 3.06 & $.27 \mathrm{e}-3$ & 3.14 \\
\hline & 4 & $.47 \mathrm{e}-1$ & 0.99 & $.61 \mathrm{e}-2$ & 2.00 & $.34389 \mathrm{e}-2$ & 2.00 & $.53 \mathrm{e}-04$ & 3.02 & $.33 \mathrm{e}-4$ & 3.04 \\
\hline & 5 & $.24 \mathrm{e}-1$ & 1.00 & $.15 \mathrm{e}-2$ & 2.00 & $.85958 \mathrm{e}-3$ & 2.00 & $.66 \mathrm{e}-05$ & 3.00 & $.41 \mathrm{e}-5$ & 3.01 \\
\hline & 6 & $.12 \mathrm{e}-1$ & 1.00 & $.38 \mathrm{e}-3$ & 2.00 & $.21489 \mathrm{e}-3$ & 2.00 & $.82 \mathrm{e}-06$ & 3.00 & $.51 \mathrm{e}-6$ & 3.00 \\
\hline \multirow{6}{*}{2} & 1 & $.89 \mathrm{e}-1$ & - & $.47 \mathrm{e}-1$ & & $.37 \mathrm{e}-1$ & - & $.44 \mathrm{e}-2$ & - & $.74982 \mathrm{e}-2$ & - \\
\hline & 2 & $.19 \mathrm{e}-1$ & 2.27 & $.50 \mathrm{e}-2$ & 3.21 & $.39 \mathrm{e}-2$ & 3.22 & $.26 \mathrm{e}-3$ & 4.09 & $.36902 \mathrm{e}-3$ & 4.34 \\
\hline & 3 & $.48 \mathrm{e}-2$ & 1.96 & $.60 \mathrm{e}-3$ & 3.08 & $.47 \mathrm{e}-3$ & 3.06 & $.16 \mathrm{e}-4$ & 3.99 & $.23515 \mathrm{e}-4$ & 3.97 \\
\hline & 4 & $.12 \mathrm{e}-2$ & 1.99 & $.74 \mathrm{e}-4$ & 3.02 & $.59 \mathrm{e}-4$ & 3.02 & $.10 \mathrm{e}-5$ & 3.99 & $.14766 \mathrm{e}-5$ & 3.99 \\
\hline & 5 & $.30 \mathrm{e}-3$ & 2.00 & $.92 \mathrm{e}-5$ & 3.00 & $.73 \mathrm{e}-5$ & 3.00 & $.64 \mathrm{e}-7$ & 4.00 & $.92393 \mathrm{e}-7$ & 4.00 \\
\hline & 6 & $.75 \mathrm{e}-4$ & 2.00 & $.12 \mathrm{e}-5$ & 3.00 & $.91 \mathrm{e}-6$ & 3.00 & $.40 \mathrm{e}-8$ & 4.00 & $.57762 \mathrm{e}-8$ & 4.00 \\
\hline
\end{tabular}

where

$$
\begin{aligned}
& T_{1}:=\left\langle\left(\widehat{\boldsymbol{q}}_{h}-\boldsymbol{q}_{h}\right) \cdot \boldsymbol{n}, u_{h}-\widehat{u}_{h}\right\rangle_{\partial \Omega_{h}}, \\
& T_{2}:=\left\langle\left(\widehat{\boldsymbol{q}}_{h}-\boldsymbol{q}_{h}\right) \cdot \boldsymbol{n}, u-\mathbb{P} u\right\rangle_{\partial \Omega_{h}}, \\
& T_{3}:=\left\langle\left(\mathrm{P}_{\partial} \boldsymbol{q}-\boldsymbol{\Pi} \boldsymbol{q}\right) \cdot \boldsymbol{n}, \widehat{u}_{h}-u_{h}\right\rangle_{\partial \Omega_{h}} .
\end{aligned}
$$

Proof. To prove this result, we begin by recalling the error equations. From the equations (1.2), it is not difficult to see that

$$
\begin{aligned}
& \left(\boldsymbol{c}\left(\boldsymbol{q}-\boldsymbol{q}_{h}\right), \boldsymbol{v}\right)_{\Omega_{h}}-\left(u-u_{h}, \nabla \cdot \boldsymbol{v}\right)_{\Omega_{h}}+\left\langle\left(u-\widehat{u}_{h}\right), \boldsymbol{v} \cdot \boldsymbol{n}\right\rangle_{\partial \Omega_{h}}=0, \\
& -\left(\left(\boldsymbol{q}-\boldsymbol{q}_{h}\right), \nabla \omega\right)_{\Omega_{h}}+\left\langle\left(\boldsymbol{q}-\widehat{\boldsymbol{q}}_{h}\right) \cdot \boldsymbol{n}, \omega\right\rangle_{\partial \Omega_{h}}=0,
\end{aligned}
$$

for all $(\boldsymbol{v}, \omega) \in \mathbf{V}_{h}^{k} \times \mathbf{W}_{h}^{k}$. As a consequence, by the definition of the projections $\boldsymbol{\Pi}$ and $\mathbb{P}$,

$$
\begin{aligned}
& \left(\boldsymbol{c}\left(\boldsymbol{q}-\boldsymbol{q}_{h}\right), \boldsymbol{v}\right)_{\Omega_{h}}-\left(\mathbb{P} u-u_{h}, \nabla \cdot \boldsymbol{v}\right)_{\Omega_{h}}+\left\langle\left(u-\widehat{u}_{h}\right), \boldsymbol{v} \cdot \boldsymbol{n}\right\rangle_{\partial \Omega_{h}}=0, \\
& -\left(\left(\boldsymbol{\Pi} \boldsymbol{q}-\boldsymbol{q}_{h}\right), \nabla \omega\right)_{\Omega_{h}}+\left\langle\left(\boldsymbol{q}-\widehat{\boldsymbol{q}}_{h}\right) \cdot \boldsymbol{n}, \omega\right\rangle_{\partial \Omega_{h}}=0,
\end{aligned}
$$

for all $(\boldsymbol{v}, \omega) \in \mathbf{V}_{h}^{k} \times \mathbf{W}_{h}^{k}$.

Thus, taking $\boldsymbol{v}=\boldsymbol{\Pi} \boldsymbol{q}-\boldsymbol{q}_{h}$ in the above equations, we get

$$
\begin{aligned}
\left\|\boldsymbol{\Pi} \boldsymbol{q}-\boldsymbol{q}_{h}\right\|_{L^{2}\left(\Omega_{h} ; \boldsymbol{c}\right)}^{2}= & \left(\boldsymbol{c}(\boldsymbol{\Pi} \boldsymbol{q}-\boldsymbol{q}), \boldsymbol{\Pi} \boldsymbol{q}-\boldsymbol{q}_{h}\right)_{\Omega_{h}}+\left(\mathbb{P} u-u_{h}, \nabla \cdot\left(\boldsymbol{\Pi} \boldsymbol{q}-\boldsymbol{q}_{h}\right)\right)_{\Omega_{h}} \\
& -\left\langle u-\widehat{u}_{h},\left(\boldsymbol{\Pi} \boldsymbol{q}-\boldsymbol{q}_{h}\right) \cdot \boldsymbol{n}\right\rangle_{\partial \Omega_{h}},
\end{aligned}
$$


TABLE 4. History of convergence for $\left.\tau\right|_{e_{K}^{\tau}}=\frac{1}{h^{2}}$ and $\left.\tau\right|_{\partial K \backslash e_{K}^{\tau}}=1$ for all $K$.

\begin{tabular}{|c|c|c|c|c|c|c|c|c|c|c|c|}
\hline \multirow[b]{2}{*}{$k$} & \multirow{2}{*}{$\begin{array}{c}\text { mesh } \\
\ell\end{array}$} & \multicolumn{2}{|c|}{$\left\|u-u_{h}\right\|_{L^{2}(\Omega)}$} & \multicolumn{2}{|c|}{$\left\|\boldsymbol{q}-\boldsymbol{q}_{h}\right\|_{L^{2}(\Omega)}$} & \multicolumn{2}{|c|}{$\left\|\boldsymbol{q}-\boldsymbol{q}_{h}^{\star}\right\|_{L^{2}(\Omega)}$} & \multicolumn{2}{|c|}{$\left\|P_{\partial} u-\widehat{u}_{h}\right\|_{L^{2}\left(\varepsilon_{h} ; h\right)}$} & \multicolumn{2}{|c|}{$\left\|u-u_{h}^{\star}\right\|_{L^{2}(\Omega)}$} \\
\hline & & error & order & error & order & error & order & error & order & error & order \\
\hline \multirow{6}{*}{0} & 1 & $.31 \mathrm{e}-0$ & - & $.15 \mathrm{e}+1$ & - & $.11 \mathrm{e}+1$ & - & $.14 \mathrm{e}-0$ & - & $.19 \mathrm{e}-0$ & - \\
\hline & 2 & $.16 \mathrm{e}-0$ & 0.98 & $.88 \mathrm{e}-0$ & 0.75 & $.68 \mathrm{e}-0$ & 0.69 & $.19 \mathrm{e}-0$ & -.42 & $.12 \mathrm{e}-0$ & 0.68 \\
\hline & 3 & $.64 \mathrm{e}-1$ & 0.72 & $.48 \mathrm{e}-0$ & 0.88 & $.38 \mathrm{e}-0$ & 0.86 & $.97 \mathrm{e}-1$ & 0.93 & $.57 \mathrm{e}-1$ & 1.02 \\
\hline & 4 & $.52 \mathrm{e}-1$ & 0.90 & $.24 \mathrm{e}-0$ & 0.96 & $.19 \mathrm{e}-0$ & 0.96 & $.49 \mathrm{e}-1$ & 0.99 & $.28 \mathrm{e}-1$ & 1.04 \\
\hline & 5 & $.26 \mathrm{e}-1$ & 0.97 & $.12 \mathrm{e}-0$ & 0.99 & $.97 \mathrm{e}-1$ & 0.99 & $.24 \mathrm{e}-1$ & 1.01 & $.13 \mathrm{e}-1$ & 1.04 \\
\hline & 6 & $.13 \mathrm{e}-1$ & 0.99 & $.62 \mathrm{e}-1$ & 1.00 & $.49 \mathrm{e}-1$ & 1.00 & $.12 \mathrm{e}-1$ & 1.00 & $.66 \mathrm{e}-2$ & 1.03 \\
\hline \multirow{6}{*}{1} & 1 & $.12 \mathrm{e}-0$ & - & $.28 \mathrm{e}-0$ & - & $.22 \mathrm{e}-0$ & - & $.52 \mathrm{e}-1$ & - & $.41 \mathrm{e}-1$ & - \\
\hline & 2 & $.25 \mathrm{e}-1$ & 2.29 & $.12 \mathrm{e}-0$ & 1.20 & $.84 \mathrm{e}-1$ & 1.38 & $.77 \mathrm{e}-2$ & 2.75 & $.56 \mathrm{e}-2$ & 2.87 \\
\hline & 3 & $.63 \mathrm{e}-2$ & 1.96 & $.32 \mathrm{e}-1$ & 1.92 & $.23 \mathrm{e}-1$ & 1.86 & $.11 \mathrm{e}-2$ & 2.88 & $.74 \mathrm{e}-3$ & 2.91 \\
\hline & 4 & $.16 \mathrm{e}-2$ & 1.97 & $.83 \mathrm{e}-2$ & 1.96 & $.59 \mathrm{e}-2$ & 1.97 & $.14 \mathrm{e}-3$ & 2.92 & $.94 \mathrm{e}-4$ & 2.98 \\
\hline & 5 & $.41 \mathrm{e}-3$ & 1.99 & $.21 \mathrm{e}-2$ & 1.98 & $.15 \mathrm{e}-2$ & 2.00 & $.18 \mathrm{e}-4$ & 2.96 & $.12 \mathrm{e}-4$ & 3.00 \\
\hline & 6 & $.10 \mathrm{e}-3$ & 1.99 & $.53 \mathrm{e}-3$ & 1.99 & $.37 \mathrm{e}-3$ & 2.00 & $.22 \mathrm{e}-5$ & 2.98 & $.15 \mathrm{e}-5$ & 3.00 \\
\hline \multirow{6}{*}{2} & 1 & $.18 \mathrm{e}-1$ & - & $.61 \mathrm{e}-1$ & - & $.52 \mathrm{e}-1$ & - & $.48 \mathrm{e}-2$ & - & $.77 \mathrm{e}-2$ & - \\
\hline & 2 & $.15 \mathrm{e}-2$ & 3.58 & $.99 \mathrm{e}-2$ & 2.61 & $.76 \mathrm{e}-2$ & 2.76 & $.47 \mathrm{e}-3$ & 3.37 & $.40 \mathrm{e}-3$ & 4.25 \\
\hline & 3 & $.21 \mathrm{e}-3$ & 2.85 & $.13 \mathrm{e}-2$ & 2.87 & $.11 \mathrm{e}-2$ & 2.82 & $.33 \mathrm{e}-4$ & 3.82 & $.26 \mathrm{e}-4$ & 3.94 \\
\hline & 4 & $.28 \mathrm{e}-4$ & 2.92 & $.18 \mathrm{e}-3$ & 2.95 & $.14 \mathrm{e}-3$ & 2.96 & $.22 \mathrm{e}-5$ & 3.93 & $.17 \mathrm{e}-5$ & 3.99 \\
\hline & 5 & $.35 \mathrm{e}-5$ & 2.97 & $.22 \mathrm{e}-4$ & 2.98 & $.17 \mathrm{e}-4$ & 2.99 & $.14 \mathrm{e}-6$ & 3.98 & $.10 \mathrm{e}-6$ & 4.00 \\
\hline & 6 & $.44 \mathrm{e}-6$ & 2.99 & $.28 \mathrm{e}-5$ & 2.99 & $.22 \mathrm{e}-5$ & 3.00 & $.86 \mathrm{e}-8$ & 4.00 & $.65 \mathrm{e}-8$ & 4.00 \\
\hline
\end{tabular}

TABLE 5 . History of convergence for when $\left.\tau\right|_{\partial K}$ takes on the values $h, 2 h, 3 h$ on the edges of $\partial K$ for all $K \in \Omega_{h}$.

\begin{tabular}{|c|c|c|c|c|c|c|c|c|c|c|c|}
\hline \multirow[b]{2}{*}{$k$} & \multirow{2}{*}{$\begin{array}{c}\text { mesh } \\
\ell\end{array}$} & \multicolumn{2}{|c|}{$\left\|u-u_{h}\right\|_{L^{2}(\Omega)}$} & \multicolumn{2}{|c|}{$\left\|\boldsymbol{q}-\boldsymbol{q}_{h}\right\|_{L^{2}(\Omega)}$} & \multicolumn{2}{|c|}{$\left\|\boldsymbol{q}-\boldsymbol{q}_{h}^{\star}\right\|_{L^{2}(\Omega)}$} & \multicolumn{2}{|c|}{$\left\|P_{\partial} u-\widehat{u}_{h}\right\|_{L^{2}\left(\varepsilon_{h} ; h\right)}$} & \multicolumn{2}{|c|}{$\left\|u-u_{h}^{\star}\right\|_{L^{2}(\Omega)}$} \\
\hline & & error & order & error & order & error & order & error & order & error & order \\
\hline \multirow{6}{*}{0} & 1 & $.67 \mathrm{e}-0$ & - & $.14 \mathrm{e}+1$ & - & $.10 \mathrm{e}+1$ & - & $.11 \mathrm{e}-0$ & - & $.16 \mathrm{e}-0$ & - \\
\hline & 2 & $.72 \mathrm{e}-0$ & $-.10 \mathrm{e}-0$ & $.66 \mathrm{e}-0$ & 1.08 & $.52 \mathrm{e}-0$ & 0.96 & $.48 \mathrm{e}-1$ & 1.24 & $.43 \mathrm{e}-1$ & 1.86 \\
\hline & 3 & $.74 \mathrm{e}-0$ & $-.45 \mathrm{e}-1$ & $.33 \mathrm{e}-0$ & 0.97 & $.26 \mathrm{e}-0$ & 1.00 & $.13 \mathrm{e}-1$ & 1.93 & $.11 \mathrm{e}-1$ & 2.03 \\
\hline & 4 & $.75 \mathrm{e}-0$ & $-.73 \mathrm{e}-2$ & $.17 \mathrm{e}-0$ & 0.99 & $.13 \mathrm{e}-0$ & 1.00 & $.48 \mathrm{e}-2$ & 1.41 & $.30 \mathrm{e}-2$ & 1.85 \\
\hline & 5 & $.75 \mathrm{e}-0$ & $.39 \mathrm{e}-3$ & $.84 \mathrm{e}-1$ & 1.00 & $.65 \mathrm{e}-1$ & 1.00 & $.23 \mathrm{e}-2$ & 1.06 & $.12 \mathrm{e}-2$ & 1.31 \\
\hline & 6 & $.75 \mathrm{e}-0$ & $.11 \mathrm{e}-2$ & $.42 \mathrm{e}-1$ & 1.00 & $.33 \mathrm{e}-1$ & 1.00 & $.11 \mathrm{e}-2$ & 1.03 & $.58 \mathrm{e}-3$ & 1.04 \\
\hline
\end{tabular}

and, after a simple integration by parts,

$$
\begin{aligned}
\left\|\boldsymbol{\Pi} \boldsymbol{q}-\boldsymbol{q}_{h}\right\|_{L^{2}\left(\Omega_{h} ; \boldsymbol{c}\right)}^{2}= & \left(\boldsymbol{c}(\boldsymbol{\Pi} \boldsymbol{q}-\boldsymbol{q}), \boldsymbol{\Pi} \boldsymbol{q}-\boldsymbol{q}_{h}\right)_{\Omega_{h}}-\left(\nabla\left(\mathbb{P} u-u_{h}\right), \boldsymbol{\Pi} \boldsymbol{q}-\boldsymbol{q}_{h}\right)_{\Omega_{h}} \\
& +\left\langle\mathbb{P} u-u_{h}-u+\widehat{u}_{h},\left(\boldsymbol{\Pi} \boldsymbol{q}-\boldsymbol{q}_{h}\right) \cdot \boldsymbol{n}\right\rangle_{\partial \Omega_{h}} .
\end{aligned}
$$


TABLE 6 . History of convergence for $\tau \equiv \frac{1}{h}$.

\begin{tabular}{|c|c|c|c|c|c|c|c|c|c|c|c|}
\hline \multirow[b]{2}{*}{$k$} & \multirow{2}{*}{$\begin{array}{c}\text { mesh } \\
\ell\end{array}$} & \multicolumn{2}{|c|}{$\left\|u-u_{h}\right\|_{L^{2}(\Omega)}$} & \multicolumn{2}{|c|}{$\left\|\boldsymbol{q}-\boldsymbol{q}_{h}\right\|_{L^{2}(\Omega)}$} & \multicolumn{2}{|c|}{$\left\|\boldsymbol{q}-\boldsymbol{q}_{h}^{\star}\right\|_{L^{2}(\Omega)}$} & \multicolumn{2}{|c|}{$\left\|P_{\partial} u-\widehat{u}_{h}\right\|_{L^{2}\left(\varepsilon_{h} ; h\right)}$} & \multicolumn{2}{|c|}{$\left\|u-u_{h}^{\star}\right\|_{L^{2}(\Omega)}$} \\
\hline & & error & order & error & order & error & order & error & order & error & order \\
\hline \multirow{6}{*}{0} & 1 & $.29 \mathrm{e}-0$ & - & $.14 \mathrm{e}+1$ & -0 & $.97 \mathrm{e}-0$ & - & $.98623 \mathrm{e}-1$ & - & $.18 \mathrm{e}-0$ & - \\
\hline & 2 & $.15 \mathrm{e}-0$ & 1.06 & $.88 \mathrm{e}-0$ & 0.68 & $.51 \mathrm{e}-0$ & 0.94 & $.23686 \mathrm{e}-0$ & -1.26 & $.14 \mathrm{e}-0$ & .32 \\
\hline & 3 & $.12 \mathrm{e}-0$ & 0.14 & $.67 \mathrm{e}-0$ & 0.37 & $.25 \mathrm{e}-0$ & 1.01 & $.23336 \mathrm{e}-0$ & $.21 \mathrm{e}-1$ & $.13 \mathrm{e}-0$ & $.12 \mathrm{e}-1$ \\
\hline & 4 & $.13 \mathrm{e}-0$ & $-.84 \mathrm{e}-2$ & $.61 \mathrm{e}-0$ & 0.14 & $.13 \mathrm{e}-0$ & 1.00 & $.23322 \mathrm{e}-0$ & $.85 \mathrm{e}-3$ & $.13 \mathrm{e}-0$ & $.26 \mathrm{e}-1$ \\
\hline & 5 & $.13 \mathrm{e}-0$ & $-.51 \mathrm{e}-2$ & $.59 \mathrm{e}-0$ & $.41 \mathrm{e}-1$ & $.63 \mathrm{e}-1$ & 1.00 & $.23323 \mathrm{e}-0$ & $-.54 \mathrm{e}-4$ & $.13 \mathrm{e}-0$ & $.63 \mathrm{e}-2$ \\
\hline & 6 & $.13 \mathrm{e}-0$ & $-.15 \mathrm{e}-2$ & $.59 \mathrm{e}-0$ & $.11 \mathrm{e}-1$ & $.31 \mathrm{e}-1$ & 1.00 & $.23323 \mathrm{e}-0$ & $-.30 e-5$ & $.13 \mathrm{e}-0$ & $.15 \mathrm{e}-2$ \\
\hline \multirow{6}{*}{1} & 1 & $.12 \mathrm{e}-0$ & - & $.32 \mathrm{e}-0$ & - & $.22 \mathrm{e}-0$ & - & $.62 \mathrm{e}-1$ & - & $.50 \mathrm{e}-01$ & - \\
\hline & 2 & $.23 \mathrm{e}-1$ & 2.35 & $.13 \mathrm{e}-0$ & 1.26 & $.85 \mathrm{e}-1$ & 1.41 & $.99 \mathrm{e}-2$ & 2.63 & $.82 \mathrm{e}-02$ & 2.61 \\
\hline & 3 & $.54 \mathrm{e}-2$ & 2.09 & $.51 \mathrm{e}-1$ & 1.37 & $.36 \mathrm{e}-1$ & 1.25 & $.23 \mathrm{e}-2$ & 2.10 & $.19 \mathrm{e}-02$ & 2.08 \\
\hline & 4 & $.13 \mathrm{e}-2$ & 2.02 & $.23 \mathrm{e}-1$ & 1.14 & $.17 \mathrm{e}-1$ & 1.08 & $.57 \mathrm{e}-3$ & 2.03 & $.48 \mathrm{e}-03$ & 2.02 \\
\hline & 5 & $.33 \mathrm{e}-3$ & 2.00 & $.11 \mathrm{e}-1$ & 1.04 & $.83 \mathrm{e}-2$ & 1.02 & $.14 \mathrm{e}-3$ & 2.01 & $.12 \mathrm{e}-03$ & 2.01 \\
\hline & 6 & $.83 e-4$ & 2.00 & $.56 \mathrm{e}-2$ & 1.01 & $.41 \mathrm{e}-2$ & 1.01 & $.36 \mathrm{e}-4$ & 2.00 & $.30 \mathrm{e}-04$ & 2.00 \\
\hline \multirow{6}{*}{2} & 1 & $.18 \mathrm{e}-01$ & - & $.45 \mathrm{e}-1$ & - & $.38 \mathrm{e}-1$ & - & $.46 \mathrm{e}-2$ & - & $.76 \mathrm{e}-2$ & - \\
\hline & 2 & $.96 \mathrm{e}-03$ & 4.19 & $.12 \mathrm{e}-1$ & 1.88 & $.76 \mathrm{e}-2$ & 2.32 & $.58 \mathrm{e}-3$ & 3.01 & $.43 \mathrm{e}-3$ & 4.16 \\
\hline & 3 & $.77 \mathrm{e}-04$ & 3.63 & $.27 \mathrm{e}-2$ & 2.16 & $.18 \mathrm{e}-2$ & 2.07 & $.64 \mathrm{e}-4$ & 3.18 & $.33 \mathrm{e}-4$ & 3.68 \\
\hline & 4 & $.77 \mathrm{e}-05$ & 3.31 & $.66 \mathrm{e}-3$ & 2.05 & $.45 \mathrm{e}-3$ & 2.01 & $.76 \mathrm{e}-5$ & 3.06 & $.31 \mathrm{e}-5$ & 3.41 \\
\hline & 5 & $.90 \mathrm{e}-06$ & 3.11 & $.16 \mathrm{e}-3$ & 2.01 & $.11 \mathrm{e}-3$ & 2.00 & $.94 \mathrm{e}-6$ & 3.01 & $.35 \mathrm{e}-6$ & 3.15 \\
\hline & 6 & $.10 \mathrm{e}-06$ & 3.03 & $.40 \mathrm{e}-4$ & 2.00 & $.28 \mathrm{e}-4$ & 2.00 & $.12 \mathrm{e}-6$ & 3.00 & $.43 \mathrm{e}-7$ & 3.04 \\
\hline
\end{tabular}

If we take $\omega=\mathbb{P} u-u_{h}$ in the second error equation and add the resulting equation, we obtain

$$
\left\|\boldsymbol{\Pi} \boldsymbol{q}-\boldsymbol{q}_{h}\right\|_{L^{2}\left(\Omega_{h} ; \boldsymbol{c}\right)}^{2}=\left(\boldsymbol{c}(\boldsymbol{\Pi} \boldsymbol{q}-\boldsymbol{q}), \boldsymbol{\Pi} \boldsymbol{q}-\boldsymbol{q}_{h}\right)_{\Omega_{h}}-T,
$$

where

$$
T:=\left\langle\left(\boldsymbol{q}-\widehat{\boldsymbol{q}}_{h}\right) \cdot \boldsymbol{n}, \mathbb{P} u-u_{h}\right\rangle_{\partial \Omega_{h}}+\left\langle u-\mathbb{P} u-\widehat{u}_{h}+u_{h},\left(\boldsymbol{\Pi} \boldsymbol{q}-\boldsymbol{q}_{h}\right) \cdot \boldsymbol{n}\right\rangle_{\partial \Omega_{h}} .
$$

It remains to show that $T=T_{1}+T_{2}+T_{3}$.

To do that, we proceed as follows. By the definition of the projection $\mathrm{P}_{\partial}$,

$$
\begin{aligned}
T= & \left\langle\left(\mathrm{P}_{\partial} \boldsymbol{q}-\widehat{\boldsymbol{q}}_{h}\right) \cdot \boldsymbol{n}, \mathbb{P} u-u_{h}\right\rangle_{\partial \Omega_{h}}+\left\langle\left(\boldsymbol{\Pi} \boldsymbol{q}-\boldsymbol{q}_{h}\right) \cdot \boldsymbol{n}, u-\mathbb{P} u\right\rangle_{\partial \Omega_{h}} \\
& +\left\langle\left(\boldsymbol{\Pi} \boldsymbol{q}-\boldsymbol{q}_{h}\right) \cdot \boldsymbol{n}, u_{h}-\widehat{u}_{h}\right\rangle_{\partial \Omega_{h}},
\end{aligned}
$$

and so,

$$
\begin{aligned}
T= & \left\langle\left(\mathrm{P}_{\partial} \boldsymbol{q}-\widehat{\boldsymbol{q}}_{h}\right) \cdot \boldsymbol{n}, \mathbb{P} u-u\right\rangle_{\partial \Omega_{h}}+\left\langle\left(\mathrm{P}_{\partial} \boldsymbol{q}-\widehat{\boldsymbol{q}}_{h}\right) \cdot \boldsymbol{n}, u-\widehat{u}_{h}\right\rangle_{\partial \Omega_{h}} \\
& +\left\langle\left(\mathrm{P}_{\partial} \boldsymbol{q}-\widehat{\boldsymbol{q}}_{h}\right) \cdot \boldsymbol{n}, \widehat{u}_{h}-u_{h}\right\rangle_{\partial \Omega_{h}} \\
& +\left\langle\left(\boldsymbol{\Pi} \boldsymbol{q}-\widehat{\boldsymbol{q}}_{h}\right) \cdot \boldsymbol{n}, u-\mathbb{P} u\right\rangle_{\partial \Omega_{h}}+\left\langle\left(\widehat{\boldsymbol{q}}_{h}-\boldsymbol{q}_{h}\right) \cdot \boldsymbol{n}, u-\mathbb{P} u\right\rangle_{\partial \Omega_{h}} \\
& +\left\langle\left(\boldsymbol{\Pi} \boldsymbol{q}-\mathrm{P}_{\partial} \boldsymbol{q}\right) \cdot \boldsymbol{n}, u_{h}-\widehat{u}_{h}\right\rangle_{\partial \Omega_{h}}+\left\langle\left(\mathrm{P}_{\partial} \boldsymbol{q}-\widehat{\boldsymbol{q}}_{h}\right) \cdot \boldsymbol{n}, u_{h}-\widehat{u}_{h}\right\rangle_{\partial \Omega_{h}} \\
& +\left\langle\left(\widehat{\boldsymbol{q}}_{h}-\boldsymbol{q}_{h}\right) \cdot \boldsymbol{n}, u_{h}-\widehat{u}_{h}\right\rangle_{\partial \Omega_{h}} .
\end{aligned}
$$


By the definition of the terms $T_{i}, i=1,2,3$,

$$
\begin{aligned}
T= & \left\langle\left(\mathrm{P}_{\partial} \boldsymbol{q}-\widehat{\boldsymbol{q}}_{h}\right) \cdot \boldsymbol{n}, \mathbb{P} u-u\right\rangle_{\partial \Omega_{h}}+\left\langle\left(\mathrm{P}_{\partial} \boldsymbol{q}-\widehat{\boldsymbol{q}}_{h}\right) \cdot \boldsymbol{n}, u-\widehat{u}_{h}\right\rangle_{\partial \Omega_{h}} \\
& +\left\langle\left(\mathrm{P}_{\partial} \boldsymbol{q}-\widehat{\boldsymbol{q}}_{h}\right) \cdot \boldsymbol{n}, \widehat{u}_{h}-u_{h}\right\rangle_{\partial \Omega_{h}}+\left\langle\left(\boldsymbol{\Pi} \boldsymbol{q}-\widehat{\boldsymbol{q}}_{h}\right) \cdot \boldsymbol{n}, u-\mathbb{P} u\right\rangle_{\partial \Omega_{h}} \\
& +T_{2}+T_{3}+\left\langle\left(\mathrm{P}_{\partial} \boldsymbol{q}-\widehat{\boldsymbol{q}}_{h}\right) \cdot \boldsymbol{n}, u_{h}-\widehat{u}_{h}\right\rangle_{\partial \Omega_{h}}+T_{1} \\
= & \left\langle\left(\mathrm{P}_{\partial} \boldsymbol{q}-\boldsymbol{\Pi} \boldsymbol{q}\right) \cdot \boldsymbol{n}, \mathbb{P} u-u\right\rangle_{\partial \Omega_{h}}+\left\langle\left(\mathrm{P}_{\partial} \boldsymbol{q}-\widehat{\boldsymbol{q}}_{h}\right) \cdot \boldsymbol{n}, u-\widehat{u}_{h}\right\rangle_{\partial \Omega_{h}} \\
& +T_{2}+T_{3}+T_{1} .
\end{aligned}
$$

The result now follows from the fact that

$$
\left\langle\left(\mathrm{P}_{\partial} \boldsymbol{q}-\boldsymbol{\Pi} \boldsymbol{q}\right) \cdot \boldsymbol{n}, \mathbb{P} u-u\right\rangle_{\partial \Omega_{h}}=0,
$$

see 9] for details, and

$$
\left\langle\left(\mathrm{P}_{\partial} \boldsymbol{q}-\widehat{\boldsymbol{q}}_{h}\right) \cdot \boldsymbol{n}, \widehat{u}_{h}-u\right\rangle_{\partial \Omega_{h}}=0,
$$

since the functions $\left(\mathrm{P}_{\partial} \boldsymbol{q}-\widehat{\boldsymbol{q}}_{h}\right) \cdot \boldsymbol{n}$ and $\widehat{u}_{h}-u$ are single-valued on all interior faces and since its product is zero on the boundary faces by $(1.2 \mathrm{e})$ and $(1.2 \mathrm{f})$. This completes the proof.

7.2. Proof of the estimate for the DG methods. By using the form of the numerical traces for the DG methods under consideration, (1.4) and (3.14), we readily obtain that

$$
\begin{aligned}
\Theta_{k}^{2} \leq & h\left(C \sum_{e \in \mathscr{E}_{h}^{i}}\left\|\llbracket \boldsymbol{q}_{h} \rrbracket\right\|_{L^{2}(e)}^{2}+\sum_{e \in \mathscr{E}_{h}^{i}}\left\|C_{11} \llbracket u_{h} \rrbracket\right\|_{L^{2}(e)}^{2}\right. \\
& +h\left(\sum_{e \in \partial \Omega_{N}}\left\|\left(\boldsymbol{q}_{h}-\boldsymbol{q}\right) \cdot \boldsymbol{n}\right\|_{L^{2}(e)}^{2}+\sum_{e \in \partial \Omega_{D}}\left\|C_{11}\left(u_{h}-u\right)\right\|_{L^{2}(e)}^{2}\right) \\
\leq & C h \max \left\{\frac{1}{\underline{C}_{22}}, \bar{C}_{11}\right\} T
\end{aligned}
$$

where

$$
\begin{aligned}
T= & \sum_{e \in \mathscr{E}_{h}^{i}}\left\|C_{22}^{1 / 2} \llbracket \boldsymbol{q}_{h} \rrbracket\right\|_{L^{2}(e)}^{2}+\sum_{e \in \mathscr{E}_{h}^{i}}\left\|C_{11}^{1 / 2} \llbracket u_{h} \rrbracket\right\|_{L^{2}(e)}^{2} \\
& +\sum_{e \in \partial \Omega_{N}}\left\|C_{22}^{1 / 2}\left(\boldsymbol{q}_{h}-\boldsymbol{q}\right) \cdot \boldsymbol{n}\right\|_{L^{2}(e)}^{2}+\sum_{e \in \partial \Omega_{D}}\left\|C_{11}^{1 / 2}\left(u_{h}-u\right)\right\|_{L^{2}(e)}^{2} \\
= & T_{1},
\end{aligned}
$$

where $T_{1}$ is defined in Lemma 7.1

To estimate $T_{1}$, we use Lemma 7.1] Thus, if we insert the expression of the numerical traces in the definitions of $T_{2}$ and $T_{3}$ and carry out some simple algebraic manipulations, we obtain

$$
\begin{aligned}
& \left|T_{2}\right| \leq \frac{1}{4} T_{1}+C\left(\bar{C}_{11}+\frac{1}{\underline{C}_{22}}\right) \sum_{K \in \Omega_{h}}\|\mathbb{P} u-u\|_{L^{2}(\partial K)}^{2}, \\
& \left|T_{3}\right| \leq \frac{1}{4} T_{1}+C\left(\bar{C}_{22}+\frac{1}{\underline{C}_{11}}\right) \sum_{K \in \Omega_{h}}\left\|\left(\mathbf{P}_{\partial} \boldsymbol{q}-\boldsymbol{\Pi} \boldsymbol{q}\right) \cdot \boldsymbol{n}\right\|_{L^{2}(\partial K)}^{2},
\end{aligned}
$$


and by Lemma 7.1, we get

$$
\begin{aligned}
T_{1} \leq & \|\boldsymbol{q}-\boldsymbol{\Pi} \boldsymbol{q}\|_{L^{2}\left(\Omega_{h}\right)}^{2}+C\left(\bar{C}_{11}+\frac{1}{\underline{C}_{22}}\right) \sum_{K \in \Omega_{h}}\|\mathbb{P} u-u\|_{L^{2}(\partial K)}^{2} \\
& +C\left(\bar{C}_{22}+\frac{1}{\underline{C}_{11}}\right) \sum_{K \in \Omega_{h}}\left\|\left(\mathbf{P}_{\partial} \boldsymbol{q}-\boldsymbol{\Pi} \boldsymbol{q}\right) \cdot \boldsymbol{n}\right\|_{L^{2}(\partial K)}^{2},
\end{aligned}
$$

and, by the approximation properties of the projections $\boldsymbol{\Pi}, \mathbb{P}$ and $\mathbf{P}_{\partial}$, 9 , we get that

where

$$
T_{1} \leq C \mathrm{C} \max \left\{\bar{C}_{11}+\frac{1}{\underline{C}_{22}}, \bar{C}_{22}+\frac{1}{\underline{C}_{11}}\right\} h^{2 k+1},
$$

$$
\mathrm{C}=|u|_{H^{k+1}\left(\Omega_{h}\right)}^{2}+|\boldsymbol{q}|_{\boldsymbol{H}^{k+1}\left(\Omega_{h}\right)}^{2}+|f|_{H^{k}\left(\Omega_{h}\right)}^{2} .
$$

As a consequence, we obtain

$$
\Theta_{k}^{2} \leq C C \max \left\{\frac{1}{\underline{C}_{22}}, \bar{C}_{11}\right\} \max \left\{\bar{C}_{11}+\frac{1}{\underline{C}_{22}}, \bar{C}_{22}+\frac{1}{\underline{C}_{11}}\right\} h^{2 k+2} .
$$

This completes the proof of Proposition 3.1

7.3. Proof of the estimate for the LDG-H methods. We begin by rewriting $\Theta_{k}^{2}$ as follows:

$$
\Theta_{k}^{2}=\Theta_{k, 1}^{2}+\Theta_{k, 2}^{2}
$$

where

$$
\begin{aligned}
& \Theta_{k, 1}^{2}=\sum_{K \in \Omega_{h}} h_{K}\left\|\left(\widehat{\boldsymbol{q}}_{h}-\boldsymbol{q}_{h}\right) \cdot \boldsymbol{n}\right\|_{L^{2}\left(\partial K \backslash e_{K}^{\tau}\right)}^{2}, \\
& \Theta_{k, 2}^{2}=\sum_{K \in \Omega_{h}} h_{K}\left\|\left(\widehat{\boldsymbol{q}}_{h}-\boldsymbol{q}_{h}\right) \cdot \boldsymbol{n}\right\|_{L^{2}\left(e_{K}^{\tau}\right)}^{2} .
\end{aligned}
$$

We are going to use Lemma 7.1 to estimate $\Theta_{k, 1}$ and a scaling argument to bound $\Theta_{k, 2}$.

Let us estimate $\Theta_{k, 1}$. We begin by noting that, by the definition of the numerical trace $\widehat{\boldsymbol{q}}_{h}$ of the LDG-H method (3.15), we have that

$$
\begin{aligned}
\Theta_{k, 1}^{2} & =\sum_{K \in \Omega_{h}} h_{K}\left\|\tau\left(\widehat{u}-u_{h}\right)\right\|_{L^{2}\left(\partial K \backslash e_{K}^{\tau}\right)}^{2} \\
& \leq C h \bar{\tau} T_{1}
\end{aligned}
$$

where

$$
T_{1}=\sum_{K \in \Omega_{h}}\left\|\tau^{1 / 2}\left(\widehat{u}_{h}-u_{h}\right)\right\|_{L^{2}(\partial K)}^{2} .
$$

Next, we estimate $T_{1}$. By using the form of the numerical traces for the LDG-H methods (3.15) and (2.8) we have that

$$
\begin{aligned}
& \left|T_{2}\right| \leq \frac{1}{4} T_{1}+\bar{\tau} \sum_{K \in \Omega_{h}}\left\|\mathbb{P} u-\mathrm{P}_{\partial} u\right\|_{L^{2}(\partial K)}^{2}, \\
& \left|T_{3}\right| \leq \frac{1}{4} T_{1}+\frac{1}{\underline{\tau}} \sum_{K \in \Omega_{h}}\left\|\left(\mathrm{P}_{\partial} \boldsymbol{q}-\boldsymbol{\Pi} \boldsymbol{q}\right) \cdot \boldsymbol{n}\right\|_{L^{2}(\partial K)}^{2} .
\end{aligned}
$$


Therefore, by Lemma 7.1 .

$$
\begin{aligned}
T_{1} \leq & \|\boldsymbol{q}-\Pi \boldsymbol{q}\|_{L^{2}\left(\Omega_{h}\right)}^{2}+\bar{\tau} \sum_{K \in \Omega_{h}}\left\|\mathbb{P} u-\mathrm{P}_{\partial} u\right\|_{L^{2}(\partial K)}^{2} \\
& +\frac{1}{\underline{\tau}} \sum_{K \in \Omega_{h}}\left\|\left(\mathrm{P}_{\partial} \boldsymbol{q}-\boldsymbol{\Pi} \boldsymbol{q}\right) \cdot \boldsymbol{n}\right\|_{L^{2}(\partial K)}^{2},
\end{aligned}
$$

and we conclude that

$$
\Theta_{k, 1}^{2} \leq C \mathrm{C}\left(\bar{\tau}^{2}+\frac{\bar{\tau}}{\underline{\tau}}\right) h^{2 k+2},
$$

where

$$
\mathrm{C}=|u|_{H^{k+1}\left(\Omega_{h}\right)}^{2}+|\boldsymbol{q}|_{\boldsymbol{H}^{k+1}\left(\Omega_{h}\right)}^{2}+|f|_{H^{k}\left(\Omega_{h}\right)}^{2} .
$$

Let us now estimate $\Theta_{k, 2}$. Using (4.16b) and (7.17) we can easily show for any $K \in \Omega_{h}$,

$$
\left(\nabla \cdot\left(\boldsymbol{q}_{h}-\boldsymbol{\Pi} \boldsymbol{q}_{h}\right), \omega\right)_{K}+\left\langle\left(\widehat{\boldsymbol{q}}_{h}-\boldsymbol{q}_{h}\right) \cdot \boldsymbol{n}-\left(\boldsymbol{\Pi} \boldsymbol{q}-\mathbf{P}_{\partial} \boldsymbol{q}\right) \cdot \boldsymbol{n}, \omega\right\rangle_{\partial K}=0,
$$

for every $\omega \in \mathcal{P}^{k}(K)$. Therefore, taking $\omega \in \mathcal{P}^{k}(K)$ so that

$$
\begin{aligned}
(\omega, v)_{K}=0, & \forall v \in \mathcal{P}^{k-1}(K), \\
\left\langle\omega-\left(\widehat{\boldsymbol{q}}_{h}-\boldsymbol{q}_{h}\right) \cdot \boldsymbol{n}, m\right\rangle_{e_{K}^{\tau}}=0, & \forall m \in \mathcal{P}^{k}\left(e_{K}^{\tau}\right),
\end{aligned}
$$

we get that

$\left\|\left(\widehat{\boldsymbol{q}}_{h}-\boldsymbol{q}_{h}\right) \cdot \boldsymbol{n}\right\|_{L^{2}\left(e_{K}^{\tau}\right)}^{2}=-\left\langle\left(\widehat{\boldsymbol{q}}_{h}-\boldsymbol{q}_{h}\right) \cdot \boldsymbol{n}, \omega\right\rangle_{\partial K \backslash e_{K}^{\tau}}+\left\langle\left(\boldsymbol{\Pi} \boldsymbol{q}-\mathbf{P}_{\partial} \boldsymbol{q}\right) \cdot \boldsymbol{n},\left(\widehat{\boldsymbol{q}}_{h}-\boldsymbol{q}_{h}\right) \cdot \boldsymbol{n}\right\rangle_{e_{K}^{\tau}}$.

Hence, since

$$
\|\omega\|_{L^{2}(\partial K)} \leq C\left\|\left(\widehat{\boldsymbol{q}}_{h}-\boldsymbol{q}_{h}\right) \cdot \boldsymbol{n}\right\|_{L^{2}\left(e_{K}^{\tau}\right)},
$$

we readily obtain

$$
\left\|\left(\widehat{\boldsymbol{q}}_{h}-\boldsymbol{q}_{h}\right) \cdot \boldsymbol{n}\right\|_{L^{2}\left(e_{K}^{\tau}\right)}^{2} \leq C\left(\left\|\left(\widehat{\boldsymbol{q}}_{h}-\boldsymbol{q}_{h}\right) \cdot \boldsymbol{n}\right\|_{L^{2}\left(\partial K \backslash e_{K}^{\tau}\right)}^{2}+\left\|\left(\boldsymbol{\Pi} \boldsymbol{q}-\mathbf{P}_{\partial} \boldsymbol{q}\right) \cdot \boldsymbol{n}\right\|_{L^{2}\left(e_{K}^{\tau}\right)}^{2}\right) .
$$

Thus, summing over $K$ gives

$$
\begin{aligned}
\Theta_{k, 2}^{2} & \leq C\left(\sum_{K \in \Omega_{h}} h_{K}\left\|\tau\left(\widehat{u}_{h}-u_{h}\right)\right\|_{L^{2}\left(\partial K \backslash e_{K}^{\tau}\right)}^{2}+\sum_{K \in \Omega_{h}} h_{K}\left\|\left(\boldsymbol{\Pi} \boldsymbol{q}-\mathbf{P}_{\partial} \boldsymbol{q}\right) \cdot \boldsymbol{n}\right\|_{L^{2}\left(e_{K}^{\tau}\right)}^{2}\right) \\
& \leq C C\left(\bar{\tau}^{2}+\frac{\bar{\tau}}{\underline{\tau}}\right) h^{2 k+2}+C|f|_{H^{k}\left(\Omega_{h}\right)}^{2} h^{2 k+2},
\end{aligned}
$$

by the approximation properties of the projection $\Pi, 9$. This completes the proof of Proposition 3.2

\section{REFERENCES}

[1] D. N. Arnold and F. Brezzi, Mixed and nonconforming finite element methods: Implementation, postprocessing and error estimates, RAIRO Modél. Math. Anal. Numér. 19 (1985), 7-32. MR813687 (87g:65126)

[2] D. N. Arnold, F. Brezzi, B. Cockburn, and L. D. Marini, Unified analysis of discontinuous Galerkin methods for elliptic problems, SIAM J. Numer. Anal. 39 (2002), 1749-1779. MR.1885715 (2002k:65183)

[3] P. Bastian and B. Rivière, Superconvergence and $H$ (div) projection for discontinuous Galerkin methods, Internat. J. Numer. Methods Fluids 42 (2003), 1043-1057. MR1991232 (2004f:65177) 
[4] C. L. Bottasso, S. Micheletti, and R. Sacco, The discontinuous Petrov-Galerkin method for elliptic problems, Comput. Methods Appl. Mech. Engng. 191 (2002), 3391-3409. MR1908187 (2003h:65153)

[5] F. Brezzi, J. Douglas, Jr., and L. D. Marini, Two families of mixed finite elements for second order elliptic problems, Numer. Math. 47 (1985), 217-235. MR799685 (87g:65133)

[6] F. Brezzi and M. Fortin, Mixed and hybrid finite element methods, Springer-Verlag, 1991. MR.1115205 (92d:65187)

[7] P. Castillo, Performance of discontinuous Galerkin methods for elliptic PDE's, SIAM J. Sci. Comput. 24 (2002), 524-547. MR1951054 (2003m:65200)

[8] P. Castillo, B. Cockburn, I. Perugia, and D. Schötzau, An a priori error analysis of the local discontinuous Galerkin method for elliptic problems, SIAM J. Numer. Anal. 38 (2000), 1676-1706. MR:1813251(2002k:65175)

[9] B. Cockburn, B. Dong, and J. Guzmán, A superconvergent LDG-hybridizable Galerkin method for second-order elliptic problems, Math. Comp., to appear.

[10] B. Cockburn, J. Gopalakrishnan, and R. Lazarov, Unified hybridization of discontinuous Galerkin, mixed and continuous Galerkin methods for second order elliptic problems. Submitted.

[11] B. Cockburn, J. Gopalakrishnan, and H. Wang, Locally conservative fluxes for the continuous Galerkin method, SIAM J. Numer. Anal. 45 (2007), 1742-1776. MR.2338408

[12] B. Cockburn, G. Kanschat, I. Perugia, and D. Schötzau, Superconvergence of the local discontinuous Galerkin method for elliptic problems on Cartesian grids, SIAM J. Numer. Anal. 39 (2001), 264-285. MR:1860725 (2002g:65140)

[13] B. Cockburn, G. Kanschat, and D. Schötzau, A locally conservative LDG method for the incompressible Navier-Stokes equations, Math. Comp. 74 (2005), 1067-1095. MR2136994 (2006a:65157)

[14] Lucia Gastaldi and Ricardo H. Nochetto, Sharp maximum norm error estimates for general mixed finite element approximations to second order elliptic equations, RAIRO Modél. Math. Anal. Numér. 23 (1989), 103-128. MR1015921 (91b:65125)

[15] P. A. Raviart and J. M. Thomas, A mixed finite element method for second order elliptic problems, Mathematical Aspects of Finite Element Methods (I. Galligani and E. Magenes, eds.), Lecture Notes in Math. 606, Springer-Verlag, New York, 1977, pp. 292-315. MR0483555 (58:3547)

[16] Rolf Stenberg, A family of mixed finite elements for the elasticity problem, Numer. Math. 53 (1988), 513-538. MR954768 (89h:65192)

[17] Rolf Stenberg, Postprocessing schemes for some mixed finite elements, RAIRO Modél. Math. Anal. Numér. 25 (1991), 151-167. MR1086845 (92a:65303)

School of Mathematics, University of Minnesota, Vincent Hall, Minneapolis, MinNESOTA 55455

E-mail address: cockburn@math.umn.edu

School of Mathematics, University of Minnesota, Vincent Hall, Minneapolis, MinNeSOTA 55455

E-mail address: guzman033@math.umn.edu

Reservoir Engineering Research Institute, 385 Sherman Avenue, Suite 5, Palo Alto, CALIFORNIA 94306

E-mail address: hywang@rerinst.org 